\title{
AN OPTIMAL-STORAGE APPROACH TO SEMIDEFINITE PROGRAMMING USING APPROXIMATE COMPLEMENTARITY
}

\author{
LIJUN DING*, ALP YURTSEVER ${ }^{\dagger}$, VOLKAN CEVHER $^{\ddagger}$, \\ JOEL A. TROPP ${ }^{\ddagger}$, AND MADELEINE UDELL ${ }^{\dagger}$
}

\begin{abstract}
This paper develops a new storage-optimal algorithm that provably solves almost all semidefinite programs (SDPs). This method is particularly effective for weakly constrained SDPs. The key idea is to formulate an approximate complementarity principle: Given an approximate solution to the dual SDP, the primal SDP has an approximate solution whose range is contained in the eigenspace with small eigenvalues of the dual slack matrix. For weakly constrained SDPs, this eigenspace has very low dimension, so this observation significantly reduces the search space for the primal solution. This result suggests an algorithmic strategy that can be implemented with minimal storage: (1) Solve the dual SDP approximately; (2) compress the primal SDP to the eigenspace with small eigenvalues of the dual slack matrix; (3) solve the compressed primal SDP. The paper also provides numerical experiments showing that this approach is successful for a range of interesting large-scale SDPs.
\end{abstract}

Key words. Semidefinite programs, Storage-optimality, Low rank, Complementary slackness, Primal recovery.

AMS subject classifications. 90C06, 90C2, 49M05

1. Introduction. Consider a semidefinite program (SDP) in the standard form

$$
\begin{array}{ll}
\text { minimize } & \operatorname{tr}(C X) \\
\text { subject to } & \mathcal{A} X=b \text { and } X \succeq 0 .
\end{array}
$$

The primal variable is the symmetric, positive-semidefinite matrix $X \in \mathbf{S}_{+}^{n}$. The problem data comprises a symmetric (but possibly indefinite) objective matrix $C \in$ $\mathbf{S}^{n}$, a righthand side $b \in \mathbf{R}^{m}$, and a linear map $\mathcal{A}: \mathbf{R}^{n \times n} \rightarrow \mathbf{R}^{m}$ with rank $m$, which can be expressed explicitly as $[\mathcal{A} H]_{i}=\operatorname{tr}\left(A_{i} H\right), i=1, \ldots, m$ for some symmetric $A_{i} \in \mathbf{S}^{n}$ and any $H \in \mathbf{R}^{n \times n}$. The notation $\operatorname{tr}(\cdot)$ stands for the trace operation: $\operatorname{tr}(A)=\sum_{i=1}^{n} a_{i i}$ for any $A \in \mathbf{R}^{n \times n}$ with $(i, j)$-th entry $a_{i j} \in \mathbf{R}$.

SDPs form a class of convex optimization problems with remarkable modeling power. But SDPs are challenging to solve because they involve a matrix variable $X \in \mathbf{S}_{+}^{n} \subset \mathbf{R}^{n \times n}$ whose dimension $n$ can rise into the millions or billions. For example, when using a matrix completion SDP in a recommender system, $n$ is the number of users and products; when using a phase retrieval SDP to visualize a biological sample, $n$ is the number of pixels in the recovered image. In these applications, most algorithms are prohibitively expensive because their storage costs are quadratic in $n$.

How much memory should be required to solve this problem? Any algorithm must be able to query the problem data and to report a representation of the solution. Informally, we say that an algorithm uses optimal storage if the working storage is no more than a constant multiple of the storage required for these operations [76]. (See Subsection 1.2 for a formal definition.)

It is not obvious how to develop storage-optimal SDP algorithms. To see why, recall that all weakly-constrained SDPs $(m=\mathcal{O}(n))$ admit low-rank solutions $[8,56]$,

\footnotetext{
* Operations Research and Information Engineering, Cornell University, Ithaca, NY, USA (ld446@cornell.edu, udell@cornell.edu).

${ }^{\dagger}$ Laboratory for Information and Inference Systems (LIONS), EPFL, Écublens, Vaud, Switzerland (alp.yurtsever@epfl.ch, volkan.cevher@epfl.ch).

${ }^{\ddagger}$ Computing and Mathematical Sciences, California Institute of Technology, Pasadena, CA, USA (jtropp@cms.caltech.edu).
} 
which can be expressed compactly in factored form. For these problems, a storageoptimal algorithm cannot even instantiate the matrix variable! One natural idea is to introduce an explicit low rank factorization of the primal variable $X$ and to minimize the problem over the factors [16].

Methods built from this idea provably work when the size of the factors is sufficiently large [12]. However, recent work [70] shows that they cannot provably solve all SDPs with optimal storage; see Section 2.

In contrast, this paper develops a new algorithm that provably solves all regular SDPs, i.e., SDPs with strong duality, unique the primal and dual solutions, and strict complementarity. These standard conditions not only hold generically [5, Definition 19], but also in many applications [45]. We defer the detailed description of these conditions in Subsection 1.1.

Our method begins with the Lagrange dual of the primal SDP (P),

$$
\begin{array}{ll}
\text { maximize } & b^{\top} y \\
\text { subject to } & C-\mathcal{A}^{\top} y \succeq 0
\end{array}
$$

with dual variable $y \in \mathbf{R}^{m}$. The vector $b^{\top}$ is the transpose of $b$, and the linear map $\mathcal{A}^{\top}: \mathbf{R}^{m} \rightarrow \mathbf{R}^{n \times n}$ is the adjoint of the linear map $\mathcal{A}$. Note the range of $\mathcal{A}^{\top}$ is in $\mathbf{S}^{n}$ because $C$ and $A_{i} \mathrm{~s}$ are symmetric. It is straightforward to compute an approximate solution to the dual SDP (D) with optimal storage using methods described in Section 6 . The challenge is to recover a primal solution from the approximate dual solution.

To meet this challenge, we develop a new approximate complementarity principle that holds for regular SDP: Given an approximate dual solution $y$, we prove that there is a primal approximate solution $X$ whose range is contained in the eigenspace with small eigenvalues of the dual slack matrix $C-\mathcal{A}^{\top} y$. This principle suggests an algorithm: we solve the primal SDP by searching over matrices with the appropriate range. This recovery problem is a (much smaller) SDP that can be solved with optimal storage.

1.1. Regularity Assumptions. First, assume that the primal (P) has a solution, say, $X_{\star}$ and the dual (D) has a unique solution $y_{\star}$. We require that strong duality holds:

$$
p_{\star}:=\operatorname{tr}\left(C X_{\star}\right)=b^{\top} y_{\star}=: d_{\star} .
$$

The condition (1.1) follows, for example, from Slater's constraint qualification.

Strong duality and feasibility imply that the solution $X_{\star}$ and the dual slack matrix $C-\mathcal{A}^{\top} y_{\star}$ satisfy the complementary slackness condition:

$$
X_{\star}\left(C-\mathcal{A}^{\top} y_{\star}\right)=0 .
$$

which implies that

$$
\operatorname{rank}\left(X_{\star}\right)+\operatorname{rank}\left(C-\mathcal{A}^{\top} y_{\star}\right) \leq n
$$

To ensure that we are not in a degenerate situation, we make the stronger assumption that every solution pair $\left(X_{\star}, y_{\star}\right)$ satisfies the stronger strict complementarity condition:

$$
\operatorname{rank}\left(X_{\star}\right)+\operatorname{rank}\left(C-\mathcal{A}^{\top} y_{\star}\right)=n .
$$

Note that these assumptions ensure that all solutions have the same rank, and therefore that the primal solution is actually unique [41, Corollary 2.5]. In particular, the rank $r_{\star}$ of the solution $X_{\star}$ satisfies the Barvinok-Pataki bound $\left(\begin{array}{c}r_{\star}+1 \\ 2\end{array}\right) \leq m$. 
To summarize, all results in this paper hold under the regularity assumptions: primal attainability, dual uniqueness, strong duality, and strict complementarity. These conditions hold generically conditioning on primal and dual attainability; i.e., for every SDP satisfying primal and dual attainability outside of a set of measure 0 [5]. The conditions or a large fraction of them are also satisfied for non-generic SDP in applications [45].

1.2. Optimal Storage. Following [76], let us quantify the storage necessary to solve every SDP (P) that satisfies our assumptions in Subsection 1.1 and that admits a solution with rank $r_{\star}$.

First, it is easy to see that $\Theta\left(n r_{\star}\right)$ numbers are sufficient to represent the rank- $r_{\star}$ solution in factored form. This cost is also necessary because every rank- $r_{\star}$ matrix is the solution to some SDP from our problem class.

To hide the internal complexity of the optimization problem (P), we will interact with the problem data using data access oracles. Suppose we can perform any of the following operations on arbitrary vectors $u, v \in \mathbf{R}^{n}$ and $y \in \mathbf{R}^{m}$ :

$$
u \mapsto C u \quad \text { and } \quad(u, v) \mapsto \mathcal{A}\left(u v^{\top}\right) \quad \text { and } \quad(u, y) \mapsto\left(\mathcal{A}^{\top} y\right) u .
$$

These oracles enjoy simple implementations in many concrete applications. The input and output of these operations clearly involve storing $\Theta(m+n)$ numbers.

In summary, any method that uses these data access oracles to solve every SDP from our class must store $\Omega\left(m+n r_{\star}\right)$ numbers. We say a method has optimal storage if the working storage provably achieves this bound.

For many interesting problems, the number $m$ of constraints is proportional to the dimension $n$. Moreover, the rank $r_{\star}$ of the solution is constant or logarithmic in $n$. In this case, a storage-optimal algorithm has working storage $\tilde{\mathcal{O}}(n)$, where the tilde suppresses log-like factors.

Remark 1.1 (Applications). The algorithmic framework we propose is most useful when the problem data has an efficient representation and the three operations in (1.4) can be implemented with low arithmetic cost. For example, it is often the case that the matrix $C$ and the linear map $\mathcal{A}$ are sparse or structured. This situation occurs in the maxcut relaxation [32], matrix completion [62], phase retrieval [21, 69], and community detection [48]. See [58] for some other examples. We expect that the assumptions mentioned in this paper (or most of them) to be satisfied for these problems. The cases of matrix completion, and community detection have been verified in [45].

1.3. From Strict Complementarity to Storage Optimality. Suppose that we have computed the exact unique dual solution $y_{\star}$. Complementary slackness (1.2) and strict complementarity (1.3) ensure that

$$
\operatorname{range}\left(X_{\star}\right) \subset \operatorname{null}\left(C-\mathcal{A}^{\top} y_{\star}\right) \quad \text { and } \quad \operatorname{dim}\left(\operatorname{null}\left(C-\mathcal{A}^{\top} y_{\star}\right)\right)=\operatorname{rank}\left(X_{\star}\right) .
$$

Therefore, the slack matrix identifies the range of the primal solution.

Let $r_{\star}$ be the rank of the primal solution. Construct an orthonormal matrix $V_{\star} \in \mathbf{R}^{n \times r_{\star}}$ whose columns span $\operatorname{null}\left(C-\mathcal{A}^{\top} y_{\star}\right)$. The compression of the primal problem $(\mathrm{P})$ to this subspace is

$$
\begin{array}{ll}
\operatorname{minimize} & \operatorname{tr}\left(C V_{\star} S V_{\star}^{\top}\right) \\
\text { subject to } & \mathcal{A}\left(V_{\star} S V_{\star}^{\top}\right)=b \quad \text { and } \quad S \succeq 0 .
\end{array}
$$


Table 1: Exact and Practical Primal Recovery

\begin{tabular}{|c|l|l|}
\hline Step & Exact Primal Recovery & Practical Primal Recovery \\
\hline 1 & Compute dual solution $y_{\star}$ & Compute approximate dual solution $y$ \\
\hline 2 & $\begin{array}{l}\text { Compute basis } V_{\star} \\
\text { for null }\left(C-\mathcal{A}^{\top} y_{\star}\right)\end{array}$ & $\begin{array}{l}\text { Compute } r_{\star} \text { eigenvectors } \\
\text { of } C-\mathcal{A}^{\top} y \text { with smallest eigenvalues; } \\
\text { collect as columns of matrix } V\end{array}$ \\
\hline 3 & Solve the compressed SDP $(1.5)$ & Solve (MinFeasSDP) \\
\hline
\end{tabular}

The variable $S \in \mathbf{S}_{+}^{r_{\star}}$ is a low-dimensional matrix when $r_{\star}$ is small. If $S_{\star}$ is a solution to (1.5), then $X_{\star}=V_{\star} S_{\star} V_{\star}^{\top}$ is a solution to the original SDP (P).

This strategy for solving the primal SDP can be implemented with a storageoptimal algorithm. Indeed, the variable $y$ in the dual SDP (D) has length $m$, so there is no obstacle to solving the dual with storage $\Theta(m+n)$ using the subgradient type method described in Section 6. We can compute the subspace $V_{\star}$ using the randomized range finder [33, Alg. 4.1] with storage cost $\Theta\left(n r_{\star}\right)$. Last, we can solve the compressed primal SDP (1.5) using working storage $\Theta\left(m+n+r_{\star}^{2}\right)$ via the matrix-free method from [25, 54]. The total storage is the optimal $\Theta\left(m+n r_{\star}\right)$. Furthermore, all of these algorithms can be implemented with the data access oracles (1.4).

Hence - assuming exact solutions to the optimization problems - we have developed a storage-optimal approach to the SDP (P), summarized in Table 1[left].

1.4. The Approximate Complementarity Principle. A major challenge remains: one very rarely has access to an exact dual solution! Rather, we usually have an approximate dual solution, obtained via some iterative dual solver.

This observation motivates us to formulate a new approximate complementarity principle. For now, assume that $r_{\star}$ is known. Given an approximate dual solution $y$, we can construct an orthonormal matrix $V \in \mathbf{R}^{n \times r_{\star}}$ whose columns are eigenvectors of $C-\mathcal{A}^{\top} y$ with the $r_{\star}$ smallest eigenvalues. Roughly speaking, the primal problem $(\mathrm{P})$ admits an approximate solution $X$ whose range is contained in $\operatorname{range}(V)$. We show the approximate solution is close to the true solution as measured in terms of suboptimality, infeasibility, and distance to the solution set.

We propose to recover the approximate primal solution by solving the semidefinite least-squares problem

(MinFeasSDP)

$\operatorname{minimize} \quad \frac{1}{2}\left\|\mathcal{A}\left(V S V^{\top}\right)-b\right\|^{2}$

subject to $\quad S \succeq 0$

with variable $S \in \mathbf{S}_{+}^{r_{\star}}$. Given a solution $\hat{S}$ to (MinFeasSDP), we obtain an (infeasible) approximate solution $X_{\text {infeas }}=V \hat{S} V^{\top}$ to the primal problem.

In fact, it is essential to relax our attention to infeasible solutions because the feasible set of $(\mathrm{P})$ should almost never contains a matrix with range $V$ ! This observation was very surprising to us, but it seems evident in retrospect. (For example, using a dimension-counting argument together with Lemma A.1.)

The resulting framework appears in Table 1[right]. This approach for solving (P) leads to storage-optimal algorithms for the same reasons described in Subsection 1.3. Our first main result ensures that this technique results in a provably good solution to the primal SDP $(\mathrm{P})$.

THEOREM 1.2 (Main theorem, informal). Instate the regularity assumptions of Sub- 
section 1.1. Suppose we have found a dual vector $y$ with suboptimality $\epsilon:=d_{\star}-b^{\top} y \leq$ const. Consider the primal reconstruction $X_{\text {infeas }}$ obtained by solving (MinFeasSDP). Then we may bound the distance between $X_{\text {infeas }}$ to the primal solution $X_{\star}$ by

$$
\left\|X_{\text {infeas }}-X_{\star}\right\|_{F}=\mathcal{O}(\sqrt{\epsilon}) .
$$

The constant in the $\mathcal{O}$ depends on the problem data $\mathcal{A}, b$, and $C$.

We state and prove the formal result as Theorem 4.1. As stated, this guarantee requires knowledge of the rank $r^{\star}$ of the solution; in Section 5, we obtain a similar guarantee using an estimate for $r^{\star}$.

1.5. Paper Organization. We discuss related work in Section 2 with a focus on storage. Section 3 contains an overview of our notation and more detailed problem assumptions. Section 4 uses the approximate complementarity principle to develop practical, robust, and theoretically justified compressed SDPs such as (MinFeasSDP) for solving $(\mathrm{P})$. These compressed SDPs are accompanied by detailed bounds on the quality of the computed solutions as compared with the true solution. Section 5 contains practical suggestions in solving these compressed SDPs such as choosing parameters, and checking the solution quality numerically. Next, we turn to algorithms for solving the dual SDP: we explain how to compute an approximate dual solution efficiently in Section 6, which provides the last ingredient for a complete method to solve (P). Section 7 shows numerically that the method is effective in practice. We conclude the paper with a discussion on contributions and future research directions in Section 8.

2. Related Work. Semidefinite programming can be traced to a 1963 paper of Bellman \& Fan [10]. Related questions emerged earlier in control theory, starting from Lyapunov's 1890 work on stability of dynamical systems. There are many classic applications in matrix analysis, dating to the 1930s. Graph theory provides another rich source of examples, beginning from the 1970s. See [13, 68, 66, 15] for more history and problem formulations.

2.1. Interior-Point Methods. The first reliable algorithms for semidefinite programming were interior-point methods (IPMs). These techniques were introduced independently by Nesterov \& Nemirovski $[52,53]$ and Alizadeh $[3,4]$.

The success of these SDP algorithms motivated new applications. In particular, Goemans \& Williamson [32] used semidefinite programming to design an approximation algorithm to compute the maximum-weight cut in a graph. Early SDP solvers could only handle graphs with a few hundred vertices [32, Sec. 5] although computational advances quickly led to IPMs that could solve problems with thousands of vertices [11].

IPMs form a series of unconstrained problems whose solutions are feasible for the original SDP, and move towards the solutions of these unconstrained problems using Newton's method. As a result, IPMs converge to high accuracy in very few iterations, but require substantial work per iteration. To solve a standard-form SDP with an $n \times n$ matrix variable and with $m$ equality constraints, a typical IPM requires $\mathcal{O}\left(\sqrt{n} \log \left(\frac{1}{\epsilon}\right)\right)$ iterations to reach a solution with accuracy $\epsilon$ (in terms of objective value) [51], and $\mathcal{O}\left(m n^{3}+m^{2} n^{2}+m^{3}\right)$ arithmetic operations per iteration (when no structure is concerned) [6], so $\mathcal{O}\left(\sqrt{n} \log \left(\frac{1}{\epsilon}\right)\left(m n^{3}+m^{2} n^{2}+m^{3}\right)\right)$ arithmetic operations in total. Further, a typical IPM requires at least $\Theta\left(n^{2}+m+m^{2}\right)$ memory not including the storage of data representation (which takes $\Theta\left(n^{2} m\right)$ memory if no structure is assumed) [6]. 
As a consequence, these algorithms are not effective for solving large problem instances, unless they enjoy a lot of structure. Hence researchers began to search for methods that could scale to larger problems.

2.2. First-Order Methods. One counterreaction to the expense of IPMs was to develop first-order optimization algorithms for SDPs. This line of work began in the late 1990s, and it accelerated as SDPs emerged in the machine learning and signal processing literature in the 2000s.

Early on, Helmberg \& Rendl [35] proposed a spectral bundle method for solving an SDP in dual form, and they showed that it converges to a dual solution when the trace of $X_{\star}$ is constant. In contrast to IPMs, the spectral bundle method has low per iteration complexity. On the other hand, the convergence rate is not known, and there is no convergence guarantee on the primal side. so there is no explicit control on the storage and arithmetic costs.

Popular first-order algorithms include the proximal gradient method [60], accelerated variants [9] and the alternating direction method of multipliers [30, 31, 14, 55]. These methods provably solve the original convex formulation of $(\mathrm{P})$. But they all store the full primal matrix variable, so they are not storage-efficient.

Recently, Friedlander \& Macedo [29] have proposed a novel first-order method that is based on gauge duality, rather than Lagrangian duality. This approach converts an SDP into an eigenvalue optimization problem. The authors propose a mechanism for using a dual solution to construct a primal solution. This paper is similar in spirit to our approach, but it lacks an analysis of the accuracy of the primal solution. Moreover, it only applies to problems with a positive-definite objective, i.e., $C \succ 0$.

2.3. Storage-Efficient First-Order Methods. Motivated by problems in signal processing and machine learning, a number of authors have revived the conditional gradient method (CGM) [28, 42]. In particular, Hazan [34] suggested using CGM for semidefinite programming. Clarkson [24] developed a new analysis, and Jaggi [37], showed how this algorithm applies to a wide range of interesting problems.

The appeal of the CGM is that it computes an approximate solution to an SDP as a sum of rank-one updates; each rank-one update is obtained from an approximate eigenvector computation. In particular, after $t$ iterations, the iterate has rank at most $t$. This property has led to the exaggeration that CGM is a "storage-efficient" optimization method when terminated early enough. Unfortunately, CGM converges very slowly, so the iterates do not have controlled rank. The literature describes many heuristics for attempting to control the rank of the iterates [57, 75], but these methods all lack guarantees.

Very recently, some of the authors of this paper [76] have shown how to use CGM to design a storage-optimal algorithm for a class of semidefinite programs by sketching the decision variable. This algorithm does not apply to standard-form SDPs, and it inherits the slow convergence of CGM. Nevertheless, the sketching methodology holds promise as a way to design storage optimal solvers, particularly together with algorithms that generalize CGM and that do apply to standard-form SDPs [74, 73].

We also mention a subgradient method developed by Renegar [59] that can be used to solve either the primal or dual SDP. Renegar's method has a computational profile similar to CGM, and it does not have controlled storage costs.

2.4. Factorization Methods. There is also a large class of heuristic SDP algorithms based on matrix factorization. The key idea is to factorize the matrix variable 
$X=F F^{\top}, F \in \mathbf{R}^{n \times r}$ and to reformulate the $\mathrm{SDP}(\mathrm{P})$ as

$$
\begin{array}{ll}
\text { minimize } & \operatorname{tr}\left(C F F^{\top}\right) \\
\text { subject to } & \mathcal{A}\left(F F^{\top}\right)=b .
\end{array}
$$

We can apply a wide range of nonlinear programming methods to optimize (2.1) with respect to the variable $F$. In contrast to the convex methods described above, these techniques only offer incomplete guarantees on storage, arithmetic, and convergence.

The factorization idea originates in the paper [36] of Homer \& Peinado. They focused on the Max-Cut SDP, and the factor $F$ was a square matrix, i.e., $r=n$. These choices result in an unconstrained nonconvex optimization problem that can be tackled with a first-order optimization algorithm.

Theoretical work of Barvinok [8] and Pataki [56] demonstrates that the primal SDP (P) always admits a solution with rank $r$, with $\left(\begin{array}{c}r+1 \\ 2\end{array}\right) \leq m$. (Note, however, that the SDP can have solutions with much lower or higher rank.)

Inspired by the existence of low rank solutions to SDP, Burer \& Monteiro [16] proposed to solve the optimization problem (2.1) where the variable $F \in \mathbf{R}^{n \times p}$ is constrained to be a tall matrix $(p \ll n)$. The number $p$ is called the factorization rank. It is clear that every rank- $r$ solution to the SDP $(\mathrm{P})$ induces a solution to the factorized problem (2.1) when $p \geq r$. Burer \& Monteiro applied a limited-memory BFGS algorithm to solve (2.1) in an explicit effort to reduce storage costs.

In subsequent work, Burer \& Monteiro [17] proved that, under technical conditions, the local minima of the nonconvex formulation (2.1) are global minima of the $\mathrm{SDP}(\mathrm{P})$, provided that the factorization rank $p$ satisfies $\left(\begin{array}{c}p+1 \\ 2\end{array}\right) \geq m+1$. As a consequence, algorithms based on (2.1) often set the factorization rank $p \approx \sqrt{2 m}$, so the storage costs are $\Omega(n \sqrt{m})$.

Unfortunately, a recent result of Waldspurger \& Walters [70, Theorem 2 \& Remark 2] demonstrates that the formulation (2.1) cannot lead to storage-optimal algorithms for interesting SDPs which are verified to be regular in [45]. In particular, suppose that the feasible set of $(\mathrm{P})$ satisfies a mild technical condition and contains a matrix with rank one. Whenever the factorization rank satisfies $\left(\begin{array}{c}p+1 \\ 2\end{array}\right)+p \leq m$, there is a set of cost matrices $C$ with positive Lebesgue measure for which the factorized problem (2.1) has (1) a unique global optimizer with rank one and (2) at least one suboptimal local minimizer, while the original SDP has a unique primal and dual solution that satisfy strict complementarity. In this situation, the variable in the factorized SDP actually requires $\Omega(n \sqrt{m})$ storage, which is not optimal if $m=\omega(1)$. In view of this negative result, we omit a detailed review of the literature on the analysis of factorization methods. See [70] for a full discussion.

3. Basics and Notation. Here we introduce some additional notation, and metrics for evaluating the quality of a solution and the conditioning of an SDP.

3.1. Notation. We will work with the Frobenius norm $\|\cdot\|_{F}$, the $\ell_{2}$ operator norm $\|\cdot\|_{\mathrm{op}}$, and its dual, the $\ell_{2}$ nuclear norm $\|\cdot\|_{*}$. We reserve the symbols $\|\cdot\|$ and $\|\cdot\|_{2}$ for the norm induced by the canonical inner product of the underlying real vector space $^{1}$.

For a matrix $B \in \mathbf{R}^{d_{1} \times d_{2}}$, we arrange its singular values in decreasing order:

$$
\sigma_{1}(B) \geq \cdots \geq \sigma_{\min \left(d_{1}, d_{2}\right)}(B) .
$$

\footnotetext{
${ }^{1}$ For symmetric matrices, we regard the trace inner product as the canonical one. For the Cartesian product $\mathbf{S}^{n} \times \mathbf{R}^{m}$, we regard the sum of trace inner product on $\mathbf{S}^{n}$ and the dot product on $\mathbf{R}^{m}$ as the canonical one.
} 
Table 2: Quality of a primal matrix $X \in \mathbf{S}_{+}^{n}$ and a dual vector $y \in \mathbf{R}^{m}$

\begin{tabular}{|c|c|c|}
\hline & primal matrix $X$ & dual vector $y$ \\
\hline suboptimality $(\epsilon)$ & $\operatorname{tr}(C X)-p_{\star}$ & $d_{\star}-b^{\top} y$ \\
\hline infeasibility $(\delta)$ & $\max \left\{\|\mathcal{A} X-b\|,\left(-\lambda_{\min }(X)\right)_{+}\right\}$ & $\left(-\lambda_{\min }(Z(y))\right)_{+}$ \\
\hline distance to solution $(d)$ & $\left\|X-X^{\star}\right\|_{\mathrm{F}}$ & $\left\|y-y_{\star}\right\|_{2}$ \\
\hline
\end{tabular}

Define $\sigma_{\min }(B)=\sigma_{\min \left(d_{1}, d_{2}\right)}(B)$ and $\sigma_{\max }(B)=\sigma_{1}(B)$. We also write $\sigma_{\min >0}(B)$ for the smallest nonzero singular value of $B$. For a linear operator $\mathcal{B}: \mathbf{S}^{d_{1}} \rightarrow \mathbf{R}^{d_{2}}$, we define

$$
\sigma_{\min }(\mathcal{B})=\min _{\|A\|=1}\|\mathcal{B}(A)\| \quad \text { and } \quad\|\mathcal{B}\|_{\text {op }}=\max _{\|A\|=1}\|\mathcal{B}(A)\|
$$

We use analogous notation for the eigenvalues of a symmetric matrix. In particular, the map $\lambda_{i}(\cdot): \mathbf{S}^{n} \rightarrow \mathbf{R}$ reports the $i$ th largest eigenvalue of its argument.

3.2. Optimal Solutions. Instate the notation and regularity assumptions from Subsection 1.1. Define the slack operator $Z: \mathbf{R}^{n} \rightarrow \mathbf{S}^{n}$ that maps a putative dual solution $y \in \mathbf{R}^{m}$ to its associated slack matrix $Z(y):=C-\mathcal{A}^{\top} y$. We omit the dependence on $y$ if it is clear from the context.

Let the rank of primal solution being $r_{\star}$ and denote its range as $\mathcal{V}_{\star}$. We also fix an orthonormal matrix $V_{\star} \in \mathbf{R}^{n \times r_{\star}}$ whose columns span $\mathcal{V}_{\star}$. Introduce the subspace $\mathcal{U}_{\star}=\operatorname{range}\left(Z\left(y_{\star}\right)\right)$, and let $U_{\star} \in \mathbf{R}^{n \times\left(n-r_{\star}\right)}$ be a fixed orthonormal basis for $\mathcal{U}_{\star}$. We have the decomposition $\mathcal{V}_{\star}+\mathcal{U}_{\star}=\mathbf{R}^{n}$.

For a matrix $V \in \mathbf{R}^{n \times r}$, define the compressed cost matrix and constraint map

$$
C_{V}:=V^{\top} C V \quad \text { and } \quad \mathcal{A}_{V}(S):=\mathcal{A}\left(V S V^{\top}\right) \text { for } S \in \mathbf{S}^{r} .
$$

In particular, $\mathcal{A}_{V_{\star}}$ is the compression of the constraint map onto the range of $X_{\star}$.

3.3. Conditioning of the SDP. Our analysis depends on conditioning properties of the pair of primal (P) and dual (D) SDPs.

First, we measure the strength of the complementarity condition (1.2) using the spectral gaps of the primal solution $X_{\star}$ and dual slack matrix $Z\left(y_{\star}\right)$ :

$$
\lambda_{\min >0}\left(X_{\star}\right) \text { and } \quad \lambda_{\min >0}\left(Z\left(y_{\star}\right)\right)
$$

These two numbers capture how far we can perturb the solutions before the complementarity condition fails.

Second, we measure the robustness of the primal solution to perturbations of the problem data $b$ using the quantity

$$
\kappa:=\frac{\sigma_{\max }(\mathcal{A})}{\sigma_{\min }\left(\mathcal{A}_{V^{\star}}\right)} .
$$

This term arises because we have to understand the conditioning of the system $\mathcal{A}_{V_{\star}}(S)=b$ of linear equations in the variable $S \in \mathbf{S}^{r_{\star}}$.

3.4. Quality of Solutions. We measure the quality of a primal matrix variable $X \in \mathbf{S}_{+}^{n}$ and a dual vector $y \in \mathbf{R}_{m}$ in terms of their suboptimality, their infeasibility, and their distance to the true solutions. Table 2 gives formulas for these quantities.

We say that a matrix $X$ is an $(\epsilon, \delta)$-solution of $(\mathrm{P})$ if its suboptimality $\epsilon_{p}(X)$ is at most $\epsilon$ and its infeasibility $\delta_{p}(X)$ is at most $\delta$. 
The primal suboptimality $\epsilon_{p}(X)$ and infeasibility $\delta_{p}(X)$ are both controlled by the distance $d_{p}(X)$ to the primal solution:

$$
\epsilon_{p}(X) \leq\|C\|_{\mathrm{F}} d_{p}(X) \quad \text { and } \quad \delta_{p}(X) \leq \max \left\{1,\|\mathcal{A}\|_{\mathrm{op}}\right\} d_{p}(X) .
$$

We can also control the distance of a dual vector $y$ and its slack matrix $Z(y)$ from their optima using the following quadratic growth lemma.

LEMMA 3.1 (Quadratic Growth). Instate the regularity assumptions from Subsection 1.1. For any dual feasible $y$ with dual slack matrix $Z(y):=C-\mathcal{A}^{\top} y$ and dual suboptimality $\epsilon=\epsilon_{d}(y)=d_{\star}-b^{\top} y$, we have

$$
\left\|(Z(y), y)-\left(Z\left(y_{\star}\right), y_{\star}\right)\right\| \leq \frac{1}{\sigma_{\min }(\mathcal{D})}\left[\frac{\epsilon}{\lambda_{\min >0}\left(X_{\star}\right)}+\sqrt{\frac{2 \epsilon}{\lambda_{\min >0}\left(X_{\star}\right)}\|Z(y)\|_{\mathrm{op}}}\right],
$$

where the linear operator $\mathcal{D}: \mathbf{S}^{n} \times \mathbf{R}^{m} \rightarrow \mathbf{S}^{n} \times \mathbf{S}^{n}$ is defined by

$$
\mathcal{D}(Z, y):=\left(Z-\left(U_{\star} U_{\star}^{\top}\right) Z\left(U_{\star} U_{\star}^{\top}\right), Z+\mathcal{A}^{\top} y\right) .
$$

The orthonormal matrix $U_{\star}$ is defined in Subsection 3.2. The quantity $\sigma_{\min }(\mathcal{D})$ is defined as $\sigma_{\min }(\mathcal{D}):=\min _{\|(Z, y)\|=1}\left\|\left(Z-\left(U_{\star} U_{\star}^{\top}\right) Z\left(U_{\star} U_{\star}^{\top}\right), Z+\mathcal{A}^{\top} y\right)\right\|$.

The proof of Lemma 3.1 can be found in Appendix A. The name quadratic growth arises from a limit of inequality (3.4): when $\epsilon$ is small, the second term in the bracket dominates the first term, so $\left\|y-y_{\star}\right\|_{2}^{2}=\mathcal{O}(\epsilon)[26]$.

4. Reduced SDPs and Approximate Complementarity. In this section, we describe two reduced SDP formulations, and we explain when their solutions are nearly optimal for the original SDP (P). We can interpret these results as constructive proofs of the approximate complementarity principle.

4.1. Reduced SDPs. Suppose that we have obtained a dual approximate solution $y$ and its associated dual slack matrix $Z(y):=C-\mathcal{A}^{\top} y$. Let $r$ be a rank parameter, which we will discuss later. Construct an orthonormal matrix $V \in \mathbf{R}^{n \times r}$ whose range is an $r$-dimensional invariant subspace associated with the $r$ smallest eigenvalues of the dual slack matrix $Z(y)$. Our goal is to compute a matrix $X$ with range $V$ that approximately solves the primal SDP (P).

Our first approach minimizes infeasibility over all psd matrices with range $V$ :

(MinFeasSDP)

$$
\begin{array}{ll}
\text { minimize } & \frac{1}{2}\left\|\mathcal{A}_{V}(S)-b\right\|^{2} \\
\text { subject to } & S \succeq 0
\end{array}
$$

with variable $S \in \mathbf{S}^{r}$. Given a solution $\hat{S}$, we can form an approximate solution $X_{\text {infeas }}=V \hat{S} V^{\top}$ for the primal SDP $(\mathrm{P})$. This is the same method from Subsection 1.4.

Our second approach minimizes the objective value over all psd matrices with range $V$, subject to a specified limit $\delta$ on infeasibility:

$$
\begin{array}{ll}
\text { (MinObjSDP) } & \text { minimize } \operatorname{tr}\left(C_{V} S\right) \\
\text { subject to } & \left\|\mathcal{A}_{V}(S)-b\right\| \leq \delta \quad \text { and } \quad S \succeq 0,
\end{array}
$$

with variable $S \in \mathbf{S}^{r}$. Given a solution $\tilde{S}$, we can form an approximate solution $X_{\mathrm{obj}}=V \tilde{S} V^{\top}$ for the primal SDP $(\mathrm{P})$. 
As we will see, both approaches lead to satisfactory solutions to the original SDP (P) under appropriate assumptions. Theorem 4.1 addresses the performance of (MinFeasSDP), while Theorem 4.6 addresses the performance of (MinObjSDP). Table 3 summarizes the hypotheses we impose to study each of the two problems, as well as the outcomes of the analysis.

The bounds in this section depend on the problem data and rely on assumptions that are not easy to check. We discuss how to check the quality of $X_{\text {infeas }}$ and $X_{\mathrm{obj}}$. in Section 5.

4.2. Analysis of (MinFeasSDP). First, we establish a result that connects the solution of (MinFeasSDP) with the solution of the original problem $(\mathrm{P})$.

THEOREM 4.1 (Analysis of (MinFeasSDP)). Instate the regularity assumptions in Subsection 1.1. Moreover, assume the solution rank $r_{\star}$ is known. Set $r=r_{\star}$. Let $y \in \mathbf{R}^{m}$ be feasible for the dual SDP (D) with suboptimality $\epsilon=\epsilon_{d}(y)=d_{\star}-b^{\top} y<c_{1}$, where the constant $c_{1}>0$ depends only on $\mathcal{A}, b$ and $C$. Then the threshold $T:=$ $\lambda_{n-r}(Z(y))$ obeys

$$
T:=\lambda_{n-r}(Z(y)) \geq \frac{1}{2} \lambda_{n-r}\left(Z\left(y_{\star}\right)\right)>0,
$$

and we have the bound

$$
\left\|X_{\text {infeas }}-X_{\star}\right\|_{F} \leq(1+2 \kappa)\left(\frac{\epsilon}{T}+\sqrt{2 \frac{\epsilon}{T}\left\|X_{\star}\right\|_{\text {op }}}\right) .
$$

This bound shows that $\left\|X_{\text {infeas }}-X_{\star}\right\|_{\mathrm{F}}^{2}=\mathcal{O}(\epsilon)$ when the dual vector $y$ is $\epsilon$ suboptimal. Notice this result requires knowledge of the solution rank $r_{\star}$. The proof of Theorem 4.1 occupies the rest of this section.

4.2.1. Primal Optimizers and the Reduced Search Space. The first step in the argument is to prove that $X_{\star}$ is near the search space $\left\{V S V^{\top}: S \in \mathbf{S}_{+}^{r}\right\}$ of the reduced problems.

LEMMA 4.2. Instate the regularity assumptions in Subsection 1.1. Further suppose $y \in \mathbf{R}^{m}$ is feasible and $\epsilon$-suboptimal for the dual SDP (D), and construct the orthonormal matrix $V$ as in Subsection 4.1. Assume that the threshold $T:=$ $\lambda_{n-r}\left(C-\mathcal{A}^{\top} y\right)>0$. Define $P_{V}(X)=V V^{\top} X V V^{\top}$, and $P_{V^{\perp}}(X)=X-P_{V}(X)$ for any $X \in \mathbf{S}^{n}$. Then for any solution $X_{\star}$ of the primal $S D P(\mathrm{P})$,

$$
\left\|P_{V^{\perp}}\left(X_{\star}\right)\right\|_{F} \leq \frac{\epsilon}{T}+\sqrt{2 \frac{\epsilon}{T}\left\|X_{\star}\right\|_{\mathrm{op}}} \text {, and }\left\|P_{V^{\perp}}\left(X_{\star}\right)\right\|_{*} \leq \frac{\epsilon}{T}+2 \sqrt{r \frac{\epsilon}{T}\left\|X_{\star}\right\|_{\mathrm{op}}} .
$$

To prove the lemma, we will utilize the following result (proved in Appendix B) which bounds the distance to subspaces via the inner product. This result might be of independent interest.

Lemma 4.3. Suppose $X, Z \in \mathbf{S}^{n}$ are both positive semidefinite. Let $V \in \mathbf{R}^{n \times r}$ be the matrices formed by the eigenvectors with the smallest $r$ eigenvalues of $Z$. Let $\epsilon=\operatorname{tr}(X Z)$ and $P_{V}(X)=V V^{\top} X V V^{\top}$. If $T=\lambda_{n-r}(Z)>0$, then

$$
\left\|X-P_{V}(X)\right\|_{F} \leq \frac{\epsilon}{T}+\sqrt{2 \frac{\epsilon}{T}\|X\|_{\mathrm{op}}} \text {, and }\left\|X-P_{V}(X)\right\|_{*} \leq \frac{\epsilon}{T}+2 \sqrt{r \frac{\epsilon}{T}\|X\|_{\mathrm{op}}}
$$

Now we are ready to prove Lemma 4.2. 
Proof of Lemma 4.2. We shall utilize Lemma 4.3. Simply set $Z$ in Lemma 4.3 to be $C-\mathcal{A}^{\top} y$ from the approximate dual solution $y$, and $X$ to be the primal solution $X_{\star}$. Using strong duality in the following step $(a)$ and feasibility of $X_{\star}$ in the following step $(b)$, we have

$$
\epsilon=b^{\top} y_{\star}-b^{\top} y \stackrel{(a)}{=} \operatorname{tr}\left(C X_{\star}\right)-b^{\top} y \stackrel{(b)}{=} \operatorname{tr}\left(C X_{\star}\right)-\left(\mathcal{A} X_{\star}\right)^{\top} y=\operatorname{tr}\left(X_{\star} Z\right) .
$$

Hence, we can apply Lemma 4.3 to obtain the bounds in Lemma 4.2.

4.2.2. Relationship between the Solutions of (MinFeasSDP) and (P). Lemma 4.2 shows that any solution $X_{\star}$ of $(\mathrm{P})$ is close to its compression $V V^{\top} X_{\star} V V^{\top}$ onto the range of $V$. Next, we show that $X_{\text {infeas }}$ is also close to $V V^{\top} X_{\star} V V^{\top}$. We can invoke strong convexity of the objective of (MinFeasSDP) to achieve this goal.

LEMma 4.4. Instate the assumptions and notation from Lemma 4.2. Assume $\sigma_{\min }\left(\mathcal{A}_{V}\right)>0$. and that the threshold $T=\lambda_{n-r}(Z(y))>0$. Then

$$
\left\|X_{\text {infeas }}-X_{\star}\right\|_{F} \leq\left(1+\frac{\sigma_{\max }(\mathcal{A})}{\sigma_{\min }\left(\mathcal{A}_{V}\right)}\right)\left(\frac{\epsilon}{T}+\sqrt{2 \frac{\epsilon}{T}\left\|X_{\star}\right\|_{\mathrm{op}}}\right),
$$

where $X_{\star}$ is any solution of the primal $S D P(\mathrm{P})$.

Proof. Since we assume that $\sigma_{\min }\left(\mathcal{A}_{V}\right)>0$, we know the objective of (MinFeasSDP), $f(S)=\frac{1}{2}\left\|\mathcal{A}_{V}(S)-b\right\|_{2}^{2}$, is $\sigma_{\min }^{2}\left(\mathcal{A}_{V}\right)$-strongly convex, and so the solution $S_{\star}$ is unique. We then have for any $S \in \mathbf{S}^{r}$

$$
\begin{aligned}
f(S)-f\left(S^{\star}\right) & \stackrel{(a)}{\geq} \operatorname{tr}\left(\nabla f\left(S^{\star}\right)^{\top}\left(S-S^{\star}\right)\right)+\frac{\sigma_{\min }^{2}\left(\mathcal{A}_{V}\right)}{2}\left\|S-S^{\star}\right\|_{\mathrm{F}}^{2} \\
& \stackrel{(b)}{\geq} \frac{\sigma_{\min }^{2}\left(\mathcal{A}_{V}\right)}{2}\left\|S-S^{\star}\right\|_{\mathrm{F}}^{2},
\end{aligned}
$$

where step $(a)$ uses strong convexity and step $(b)$ is due to the optimality of $S_{\star}$.

Since $\mathcal{A} X_{\star}=b$, we can bound the objective of (MinFeasSDP) by $\left\|P_{V}(X)-X_{\star}\right\|_{F}$ :

$$
\left\|\mathcal{A}_{V}\left(V^{\top} X V\right)-b\right\|_{2}=\left\|\mathcal{A}\left(P_{V}(X)-X_{\star}\right)\right\|_{2} \leq \sigma_{\max }(\mathcal{A})\left\|P_{V}(X)-X_{\star}\right\|_{\mathrm{F}} .
$$

Combining pieces, we know that $S_{\star}$ satisfies

$$
\begin{aligned}
\left\|S^{\star}-V^{\top} X_{\star} V\right\|_{\mathrm{F}}^{2} & \stackrel{(a)}{\leq} \frac{2}{\sigma_{\min }^{2}\left(\mathcal{A}_{V}\right)}\left(f\left(V^{\top} X_{\star} V\right)-f\left(S^{\star}\right)\right) \stackrel{(b)}{\leq} \frac{\sigma_{\max }^{2}(\mathcal{A})}{\sigma_{\min }^{2}\left(\mathcal{A}_{V}\right)}\left\|X_{\star}-P_{V}\left(X_{\star}\right)\right\|_{\mathrm{F}}^{2} \\
& \stackrel{(c)}{\leq} \frac{\sigma_{\max }^{2}(\mathcal{A})}{\sigma_{\min }^{2}\left(\mathcal{A}_{V}\right)}\left(\frac{\epsilon}{T}+\sqrt{2 \frac{\epsilon}{T}\left\|X_{\star}\right\|_{\mathrm{op}}}\right)^{2}
\end{aligned}
$$

where step (a) uses (4.3), step (b) uses (4.4) for $X=X_{\star}$ and $f\left(S^{\star}\right) \geq 0$, and step $(c)$ uses Lemma 4.2. Lifting to the larger space $\mathbf{R}^{n \times n}$, we see

$$
\begin{aligned}
\left\|V S^{\star} V^{\top}-X_{\star}\right\|_{\mathrm{F}} & \leq\left\|V S^{\star} V^{\top}-P_{V}\left(X_{\star}\right)\right\|_{\mathrm{F}}+\left\|X_{\star}-P_{V}(X)\right\|_{\mathrm{F}} \\
& \stackrel{(a)}{=}\left\|S^{\star}-V^{\top} X_{\star} V\right\|_{\mathrm{F}}+\left\|X_{\star}-P_{V}\left(X_{\star}\right)\right\|_{\mathrm{F}} \\
& \stackrel{(b)}{\leq}\left(1+\frac{\sigma_{\max }(\mathcal{A})}{\sigma_{\min }\left(\mathcal{A}_{V}\right)}\right)\left(\frac{\epsilon}{T}+\sqrt{2 \frac{\epsilon}{T}\left\|X_{\star}\right\|_{\mathrm{op}}}\right) .
\end{aligned}
$$

Here we use the unitary invariance of $\|\cdot\|_{\mathrm{F}}$ in $(a)$. The inequality $(b)$ is due to our bound above for $S_{\star}$ and Lemma 4.2. 
4.2.3. Lower Bounds for the Threshold and Minimum Singular Value. Finally, we must confirm that the extra hypotheses of Lemma 4.4 hold, i.e., $T>0$ and $\sigma_{\min }\left(\mathcal{A}_{V}\right)>0$.

We explain the intuition here. Strict complementarity forces $\lambda_{n-r}\left(Z\left(y_{\star}\right)\right)>0$. If $Z$ is close to $Z\left(y_{\star}\right)$, then we expect that $T>0$ by continuity. When $X_{\star}$ is unique, Lemma A.1 implies that $\operatorname{null}\left(\mathcal{A}_{V_{\star}}\right)=\{0\}$. As a consequence, $\sigma_{\min }\left(\mathcal{A}_{V_{\star}}\right)>0$. If $V$ is close to $V_{\star}$, then we expect that $\sigma_{\min }\left(\mathcal{A}_{V}\right)>0$ as well. We have the following rigorous statement.

LEMma 4.5. Instate the hypotheses of Theorem 4.1. Then

$$
\begin{aligned}
T=\lambda_{n-r}(Z(y)) & \geq \frac{1}{2} \lambda_{n-r}\left(Z\left(y_{\star}\right)\right) ; \\
\sigma_{\min }\left(\mathcal{A}_{V}\right) & \geq \frac{1}{2} \sigma_{\min }\left(\mathcal{A}_{V^{\star}}\right)>0 .
\end{aligned}
$$

Proof. We first prove the lower bound on the threshold $T$. Using $\|(Z, y)-$ $\left(Z\left(y_{\star}\right), y_{\star}\right)\|\geq\| Z-Z\left(y_{\star}\right)\left\|_{\mathrm{op}} \geq\right\| Z\left\|_{\mathrm{op}}-\right\| Z\left(y_{\star}\right) \|_{\mathrm{op}}$ and quadratic growth (Lemma 3.1) we have

$$
\|Z\|_{\mathrm{op}}-\left\|Z\left(y_{\star}\right)\right\|_{\mathrm{op}} \leq \frac{1}{\sigma_{\min }(\mathcal{D})}\left(\frac{\epsilon}{\lambda_{\min >0}\left(X_{\star}\right)}+\sqrt{\frac{2 \epsilon}{\lambda_{\min >0}\left(X_{\star}\right)}\|Z\|_{\mathrm{op}}}\right) .
$$

Thus for sufficiently small $\epsilon$, we have $\|Z\|_{\text {op }} \leq 2\left\|Z\left(y_{\star}\right)\right\|_{\text {op }}$. Substituting this bound into previous inequality gives,

$$
\left\|(Z, y)-\left(Z\left(y_{\star}\right), y_{\star}\right)\right\| \leq \frac{1}{\sigma_{\min }(\mathcal{D})}\left(\frac{\epsilon}{\lambda_{\min >0}\left(X_{\star}\right)}+\sqrt{\frac{4 \epsilon}{\lambda_{\min >0}\left(X_{\star}\right)}\left\|Z\left(y_{\star}\right)\right\|_{\mathrm{op}}}\right) .
$$

Weyl's inequality tells us that $\lambda_{n-r}\left(Z\left(y_{\star}\right)\right)-T \leq\left\|Z-Z\left(y_{\star}\right)\right\|_{\text {op }}$. Using (4.5), we see that for all sufficiently small $\epsilon, T:=\lambda_{n-r}\left(C-\mathcal{A}^{\top} y\right) \geq \frac{1}{2} \lambda_{n-r}\left(Z\left(y_{\star}\right)\right)$.

Next we prove the lower bound on $\mathcal{A}_{V}$. We have $\sigma_{\min }\left(\mathcal{A}_{V^{\star}}\right)>0$ by Lemma A.1. It will be convenient to align the columns of $V$ with those of $V^{\star}$ for our analysis. Consider the solution $O_{\star}$ to the orthogonal Procrustes problem $O_{\star}=\operatorname{argmin} O^{\top}=I, O \in \mathbf{R}^{r \times r}\left\|V O-V^{\star}\right\|_{\mathrm{F}}$. Since $\sigma_{\min }\left(\mathcal{A}_{V}\right)=\sigma_{\min }\left(\mathcal{A}_{V O_{\star}}\right)$ for orthonormal $O_{\star}$, without loss of generality, we suppose we have already performed the alginment and $V$ is $V O_{\star}$ in the following.

Let $S_{1}=\operatorname{argmin}_{\|S\|_{\mathrm{F}}=1}\left\|\mathcal{A}_{V}(S)\right\|_{2}$. Then we have

$$
\begin{aligned}
\sigma_{\min }\left(\mathcal{A}_{V_{\star}}\right)-\sigma_{\min }\left(\mathcal{A}_{V}\right) & \leq\left\|\mathcal{A}_{V_{\star}}\left(S_{1}\right)\right\|_{2}-\left\|\mathcal{A}_{V}\left(S_{1}\right)\right\|_{2} \\
& \leq\left\|\mathcal{A}\left(V^{\star} S_{1}\left(V^{\star}\right)^{\top}\right)-\mathcal{A}\left(V S_{1} V^{\top}\right)\right\|_{2} \\
& \leq\|\mathcal{A}\|_{\mathrm{op}}\left\|V^{\star} S_{1}\left(V^{\star}\right)^{\top}-\left(V S_{1} V^{\top}\right)\right\|_{\mathrm{F}} .
\end{aligned}
$$

Defining $E=V-V^{\star}$, we bound the term $\left\|V^{\star} S_{1}\left(V^{\star}\right)^{\top}-\left(V S_{1} V^{\top}\right)\right\|_{\mathrm{F}}$ as

$$
\begin{aligned}
\left\|V^{\star} S_{1}\left(V^{\star}\right)^{\top}-\left(V S_{1} V^{\top}\right)\right\|_{\mathrm{F}} & =\left\|E S_{1}\left(V_{\star}\right)^{\top}+V_{\star} S_{1} E^{\top}+E S_{1} E^{\top}\right\|_{\mathrm{F}} \\
& \stackrel{(a)}{\leq} 2\|E\|_{\mathrm{F}}\left\|V_{\star} S_{1}\right\|_{\mathrm{F}}+\|E\|_{\mathrm{F}}^{2}\left\|S_{1}\right\|_{\mathrm{F}} \\
& \stackrel{(b)}{=} 2\|E\|_{\mathrm{F}}+\|E\|_{\mathrm{F}}^{2},
\end{aligned}
$$

where $(a)$ uses the triangle inequality and the submultiplicativity of the Frobenius norm. We use the orthogonality of the columns of $V$ and of $V^{\star}$ and the fact that $\left\|S_{1}\right\|_{\mathrm{F}}=1$ in step $(b)$. 
A variant of the Davis-Kahan inequality [72, Theorem 2] asserts that $\|E\|_{\mathrm{F}} \leq$ $4\left\|Z-Z\left(y_{\star}\right)\right\|_{\mathrm{F}} / \lambda_{\min >0}\left(Z\left(y_{\star}\right)\right)$. Combining this fact with inequality (4.5), we see $\|E\|_{\text {op }} \rightarrow 0$ as $\epsilon \rightarrow 0$. Now using (4.7) and (4.6), we see that for all sufficiently small $\epsilon, \sigma_{\min }\left(\mathcal{A}_{V}\right) \geq \frac{1}{2} \sigma_{\min }\left(\mathcal{A}_{V^{\star}}\right)>0$.

4.2.4. Proof of Theorem 4.1. Instate the hypotheses of Theorem 4.1. Now, Lemma 4.5 implies that $\sigma_{\min }\left(\mathcal{A}_{V}\right)>0$ and that $T>0$. Therefore, we can invoke Lemma 4.4 to obtain the stated bound on $\left\|X_{\text {infeas }}-X_{\star}\right\|_{\mathrm{F}}$.

4.3. Analysis of (MinObjSDP). Next, we establish a result that connects the solution to (MinObjSDP) with the solution to the original problem (P).

TheOREM 4.6 (Analysis of (MinObjSDP)). Instate the regularity assumptions in Subsection 1.1. Moreover, assume $r \geq r_{\star}$. Let $y \in \mathbf{R}^{m}$ be feasible for the dual $S D P$ (D) with suboptimality $\epsilon=\epsilon_{d}(y)=d_{\star}-b^{\top} y<c_{2}$, where the constant $c_{2}>0$ depends only on $\mathcal{A}, b$ and $C$. Then the threshold $T:=\lambda_{n-r}(Z(y))$ obeys

$$
T:=\lambda_{n-r}(Z(y)) \geq \frac{1}{2} \lambda_{n-r}\left(Z\left(y_{\star}\right)\right)>0 .
$$

Introduce the quantities

$$
\begin{aligned}
& \delta_{0}:=\sigma_{\max }(\mathcal{A})\left(\frac{\epsilon}{T}+\sqrt{2 \frac{\epsilon}{T}\left\|X_{\star}\right\|_{\mathrm{op}}}\right) \\
& \epsilon_{0}:=\min \left\{\|C\|_{F}\left(\frac{\epsilon}{T}+\sqrt{2 \frac{\epsilon}{T}\left\|X_{\star}\right\|_{\mathrm{op}}}\right),\|C\|_{\mathrm{op}}\left(\frac{\epsilon}{T}+\sqrt{2 \frac{r \epsilon}{T}\left\|X_{\star}\right\|_{\mathrm{op}}}\right)\right\} .
\end{aligned}
$$

If we solve (MinObjSDP) with the infeasibility parameter $\delta=\delta_{0}$, then the resulting matrix $X_{o b j}$ is an $\left(\epsilon_{0}, \delta_{0}\right)$ solution to $(\mathrm{P})$.

If in addition $C=I$, then $X_{o b j}$ is superoptimal with $0 \geq \epsilon_{0} \geq-\frac{\epsilon}{T}$.

The analysis Theorem 4.1 of (MinFeasSDP) requires knowledge of the solution rank $r_{\star}$, and the bounds depend on the conditioning $\kappa$. In contrast, Theorem 4.6 does not require knowledge of $r_{\star}$, and the bounds do not depend on $\kappa$. Table 3 compares our findings for the two optimization problems Theorem 4.1 and Theorem 4.6.

Remark 4.7. The quality of the primal reconstruction depends on the ratio between the threshold $T$ and the suboptimality $\epsilon$. The quality improves as the suboptimality $\epsilon$ decreases, so the primal reconstruction approaches optimality as the dual estimate $y$ approaches optimality. The threshold $T$ is increasing in the rank estimate $r$, and so the primal reconstruction improves as $r$ increases. Since $r$ controls the storage required for the primal reconstruction, we see that the quality of the primal reconstruction improves as our storage budget increases.

Remark 4.8. Using the concluding remarks of [64], the above bound on suboptimality and infeasibility shows that the distance between $X_{\mathrm{obj}}$ and $X_{\star}$ is at most $\mathcal{O}\left(\epsilon^{1 / 4}\right)$. Here, the $\mathcal{O}(\cdot)$ notation omits constants depending on $\mathcal{A}, b$, and $C$.

The proof of Theorem 4.6 occupies the rest of this subsection.

4.3.1. Bound on the Threshold via Quadratic Growth. We first bound $T$ when the suboptimality of $y$ is bounded. This bound is a simple consequence of quadratic growth (Lemma 3.1).

Lemma 4.9. Instate the hypotheses of Theorem 4.6. Then

$$
T:=\lambda_{n-r}(Z(y)) \geq \frac{1}{2} \lambda_{n-r}\left(Z\left(y_{\star}\right)\right)>0 .
$$


Table 3: Comparison of (MinFeasSDP) and (MinObjSDP) given a feasible $\epsilon$ suboptimal dual vector $y$.

\begin{tabular}{|c|c|c|}
\hline Assumption and Quality & (MinFeasSDP) & (MinObjSDP) \\
\hline \hline Require $r=r_{\star} ?$ & Yes & No \\
\hline Suboptimality & $\mathcal{O}(\kappa \sqrt{\epsilon})$ & $\mathcal{O}(\sqrt{\epsilon})$ \\
\hline Infeasibility & $\mathcal{O}(\kappa \sqrt{\epsilon})$ & $\mathcal{O}(\sqrt{\epsilon})$ \\
\hline Distance to the solution & $\mathcal{O}(\kappa \sqrt{\epsilon})$ & Remark 4.8 \\
\hline
\end{tabular}

Proof. The proof follows exactly the same line (without even changing of the notation) as the proof of Lemma 4.5 in assuring $\lambda_{n-r_{\star}}(Z(y))>0$, by noting $\lambda_{n-r}\left(Z\left(y_{\star}\right)\right)>$ 0 whenever $r \geq n-\operatorname{rank}\left(Z\left(y_{\star}\right)\right)$.

4.3.2. Proof of Theorem 4.6. Lemma 4.2 shows that any primal solution $X_{\star}$, is close to $V V^{\top} X_{\star} V V^{\top}=: P_{V}\left(X_{\star}\right)$. We must ensure that $P_{V}\left(X_{\star}\right)$ is feasible for (MinObjSDP). This is achieved by setting the infeasibility parameter in (MinObjSDP) as

$$
\delta:=\sigma_{\max }(\mathcal{A})\left(\frac{\epsilon}{T}+\sqrt{2 \frac{\epsilon\left\|X_{\star}\right\|_{\mathrm{op}}}{T}}\right)
$$

This choice also guarantees all solutions to (MinObjSDP) are $\delta$-feasible.

The solution to (MinObjSDP) is $\delta_{0}$-feasible by construction. It remains to show the solution is $\epsilon_{0}$-suboptimal. We can bound the suboptimality of the feasible point $P_{V}\left(X_{\star}\right)$ to produce a bound on the suboptimality of the solution to (MinObjSDP). We use Hölder's inequality to translate the bound on the distance between $P_{V}\left(X_{\star}\right)$ and $X_{\star}$, from Lemma 4.2, into a bound on the suboptimality:

$$
\begin{aligned}
& \operatorname{tr}\left(C\left(P_{V}\left(X_{\star}\right)-X_{\star}\right)\right) \\
& \leq \epsilon_{0}:=\min \left\{\|C\|_{\mathrm{F}}\left(\frac{\epsilon}{T}+\sqrt{2 \frac{\epsilon}{T}\left\|X_{\star}\right\|_{\mathrm{op}}}\right),\|C\|_{\mathrm{op}}\left(\frac{\epsilon}{T}+\sqrt{2 \frac{r \epsilon}{T}\left\|X_{\star}\right\|_{\mathrm{op}}}\right)\right\} .
\end{aligned}
$$

This argument shows that $P_{V}\left(X_{\star}\right)$ is feasible, and hence the solution to (MinObjSDP), is at most $\epsilon_{0}$ suboptimal.

To prove the improvement for the case $C=I$, we first complete $V$ to form a basis $W=[U V]$ for $\mathbf{R}^{n}$, where $U=\left[v_{r+1}, \ldots, v_{n}\right] \in \mathbf{R}^{n \times(n-r)}$ and where $v_{i}$ is the eigenvector of $Z$ associated with the $i$-th smallest eigenvalue. Define $X_{1}=U^{\top} X_{\star} U$ and $X_{2}=V^{\top} X_{\star} V$. We first note that

$$
\operatorname{tr}\left(X_{\star}\right)=\operatorname{tr}\left(W^{\top} X_{\star} W\right)=\operatorname{tr}\left(X_{1}\right)+\operatorname{tr}\left(X_{2}\right), \quad \text { and } \quad \operatorname{tr}\left(X_{2}\right)=\operatorname{tr}\left(V V^{\top} X_{\star} V V^{\top}\right) .
$$

We can bound $\operatorname{tr}\left(X_{1}\right)$ using the following inequality:

$$
\epsilon \stackrel{(a)}{=} \operatorname{tr}\left(Z X_{\star}\right)=\sum_{i=1}^{n} \lambda_{n-i+1}(Z) v_{i}^{\top} X_{\star} v_{i} \stackrel{(b)}{\geq} T \sum_{i=r+1}^{n} v_{i}^{\top} X_{\star} v_{i}=\operatorname{tr}\left(U X_{\star} U^{\top}\right)=\operatorname{tr}\left(X_{1}\right) .
$$

Here step $(a)$ is due to strong duality and we uses $v_{i}^{\top} X_{\star} v_{i} \geq 0$ in step $(b)$ as $X_{\star} \succeq 0$. Combing pieces and $\operatorname{tr}\left(X_{1}\right) \geq 0$ as $X_{1} \succeq 0$, we find that

$$
\operatorname{tr}\left(X_{\star}\right) \geq \operatorname{tr}\left(V V^{\top} X_{\star} V V^{\top}\right) \geq \operatorname{tr}\left(X_{\star}\right)-\frac{\epsilon}{T} .
$$

This completes the argument. 


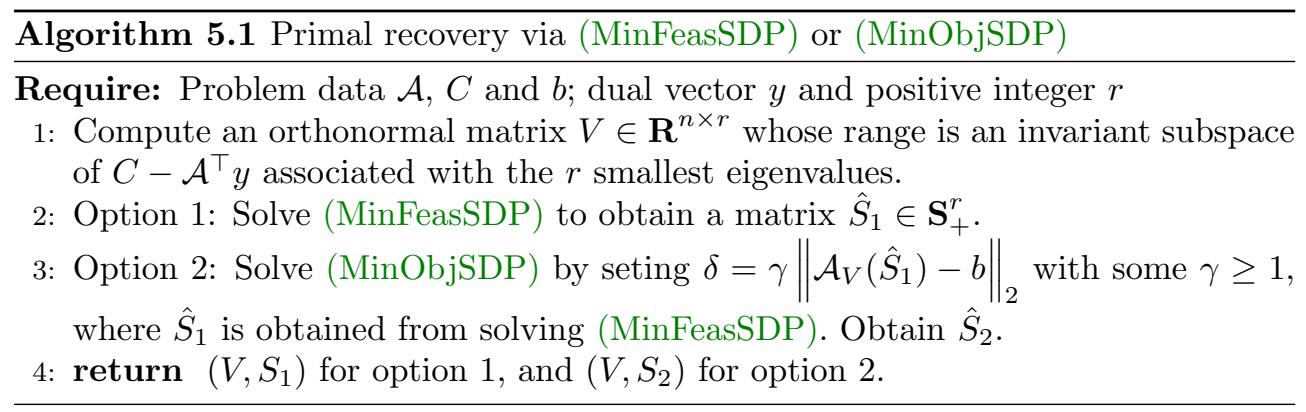

Table 4: Required operators for solving MinFeasSDP and MinObjSDP. The operators $P_{\mathbf{B}_{\delta}}$ and $P_{\mathbf{S}_{+}^{r}}$ are projections of the $\ell_{2}$ norm ball $\mathbf{B}_{\delta}:=\left\{y \in \mathbf{R}^{m} \mid\|y\|_{2} \leq \delta\right\}$ and of the PSD matrices of side dimension $r, \mathbf{S}_{+}^{r}$, respectively.

\begin{tabular}{|c|c|c|c|c|}
\hline Operator & Input & Output & Storage req. & Time Compl. \\
\hline $\mathcal{A}_{V}$ & $S \in \mathbf{S}^{r}$ & $\mathcal{A}_{V}(S) \in \mathbf{R}^{m}$ & $r^{2}+m$ & $\mathcal{O}\left(r^{2} L_{\mathcal{A}}\right)$ \\
$\mathcal{A}_{V}^{\top}$ & $y \in \mathbf{R}^{m}$ & $V^{\top}\left(\mathcal{A}^{\top}(y)\right) V \in \mathbf{S}^{r}$ & $m+r^{2}$ & $\mathcal{O}\left(r L_{\left.\mathcal{A}^{\top}+n r^{2}\right)}\right.$ \\
$P_{\mathbf{B}_{\delta}}$ & $y \in \mathbf{R}^{m}$ & $P_{\mathbf{B}_{\delta}}(y) \in \mathbf{R}^{m}$ & $2 m$ & $\mathcal{O}(m)$ \\
$P_{\mathbf{S}_{+}^{r}}$ & $S \in \mathbf{S}^{r}$ & $P_{\mathbf{S}_{+}^{r}}(S) \in \mathbf{S}^{r}$ & $2 r^{2}$ & $\mathcal{O}\left(r^{3}\right)$ \\
\hline
\end{tabular}

5. Computational Aspects of Primal Recovery. The previous section introduced two methods, (MinFeasSDP) and (MinObjSDP), to recover an approximate primal from an approximate dual solution $y$. It contains theoretical bounds on suboptimality, infeasibility, and distance to the solution set of the primal SDP (P). We summarize this approach as Algorithm 5.1.

In this section, we turn this approach into a practical optimal storage algorithm, by answering the following questions:

1. How should we solve (MinFeasSDP) and (MinObjSDP)?

2. How should we choose $\delta$ in (MinObjSDP)?

3 . How should we choose the rank parameter $r$ ?

4. How can we estimate the suboptimality, infeasibility, and (possibly) the distance to the solution to use as stopping conditions?

In particular, our choices for algorithmic parameters should not depend on any quantities that are unknown or difficult to compute. We address each question in turn.

For this discussion, let us quantify the cost of the three data access oracles (1.4). We use the mnemonic notation $L_{C}, L_{\mathcal{A}}$, and $L_{\mathcal{A}^{\top}}$ for the respective running time (denominated in flops) of the three operations.

5.1. Solving MinFeasSDP and MinObjSDP. Suppose that we have a dual estimate $y \in \mathbf{R}^{m}$, and that we have chosen $r=\mathcal{O}\left(r_{\star}\right)$ and $\delta$. Each recovery problem, (MinFeasSDP) and (MinObjSDP), is an SDP with an $r \times r$ decision variable and $m$ linear constraints. We now discuss how to solve them with optimal storage $\mathcal{O}(m+n r)$. First, we present four operators that form the computational core of all the storage optimal algorithms we consider here. We list their input and output dimension, storage requirement (sum of input output dimensions), time complexity in evaluating these operators in Table 4. Any algorithm that uses a constant number of calls to these operators at each iteration (and at most $\mathcal{O}(m+n r)$ additional storage) achieves optimal storage $\mathcal{O}(m+n r)$. To be concrete, we describe algorithms 
to solve (MinFeasSDP) and (MinObjSDP) that achieve optimal storage. Many other algorithmic choices are possible.

- For (MinFeasSDP), we can use the accelerated projected gradient method [51]. This method uses the operators $\mathcal{A}_{V}, \mathcal{A}_{V}^{\top}$, and $P_{\mathrm{S}_{+}^{r}}$. Each iteration requires one call each to $\mathcal{A}_{V}, \mathcal{A}_{V}^{\top}$, and $P_{\mathbf{S}_{+}^{r}}$, and a constant number of additions in $\mathbf{R}^{m}$ and $\mathbf{S}_{+}^{r}$. Hence the per iteration flop count is $\mathcal{O}\left(r^{2} L_{\mathcal{A}}+r L_{\mathcal{A}^{\top}}+\right.$ $\left.m+r^{2} n\right)$. As for storage, the accelerated projected gradient method requires $\mathcal{O}\left(m+r^{2}\right)$ working memory to store the residual $\mathcal{A}_{V}(S)-b$, the computed gradient, and iterates of size $r^{2}$. Hence this method is storage optimal.

- For (MinObjSDP), we can use the Chambolle-Pock method [22]. We present a detailed description in Appendix C. This method requires access to the operators $\mathcal{A}_{V}, \mathcal{A}_{V}^{\top}, P_{\mathbf{B}_{\delta}}$ and $P_{\mathbf{S}_{+}^{r}}$. It also stores the matrix $C_{V}=V^{T} C V \in$ $\mathbf{R}^{r \times r}$ explicitly. We can compute $C_{V}$ in $r^{2} L_{C}$ time and store it using $r^{2}$ storage. Each iteration requires one call each to $\mathcal{A}_{V}, \mathcal{A}_{V}^{\top}, P_{\mathbf{B}_{\delta}}$, and $P_{\mathbf{S}_{+}^{r}}$, and a constant number of additions in $\mathbf{R}^{m}$ and $\mathbf{S}_{+}^{r}$. Hence the per iteration flop count is $\mathcal{O}\left(r^{2} L_{\mathcal{A}}+r L_{\mathcal{A}^{\top}}+m+r^{2} n\right)$. As for storage, the Chambolle-Pock method requires $\mathcal{O}\left(m+r^{2}\right)$ working memory to store the residual $\mathcal{A}_{V}(S)-b$, one dual iterate of size $m$, two primal iterates of size $r^{2}$, and a few intermediate quantities of size $r^{2}$ or $m$. Hence the method is again storage-optimal.

5.2. Choosing the Rank Parameter $r$. Theorem 4.1 shows that (MinFeasSDP) recovers the solution when the rank estimate $r$ is accurate. Alas, as $r$ increases, (MinFeasSDP) can have multiple solutions. Hence it is important to use information about the objective function as well (e.g., using (MinObjSDP)) to recover the solution to $(\mathrm{P})$ - in theory. In practice, we find that (MinFeasSDP) recovers the primal solution well so long as $r$ satisfies the Barvinok-Pataki bound $\frac{r(r+1)}{2} \leq m$.

Theorem 4.6 shows that (MinObjSDP) is more robust, and provides useful results so long as the rank estimate $r$ exceeds the true rank $r_{\star}$. Indeed, the quality of the solution improves as $r$ increases. A user seeking the best possible solution to (MinObjSDP) should choose the largest rank estimate $r$ for which the SDP (MinObjSDP) can still be solved, given computational and storage limitations.

It is tempting to consider the spectrum of the dual slack matrix $C-\mathcal{A}^{\top} y$, and in particular, its smallest eigenvalues, to guess the true rank of the solution. We do not know of any reliable rules that use this idea.

5.3. Choosing the Infeasibility Parameter $\delta$. To solve (MinObjSDP), we must choose a bound $\delta$ on the acceptable infeasibility. (Recall that (MinObjSDP) is generally not feasible when $\delta=0$.) This bound can be chosen using the result of (MinFeasSDP). Concretely, solve (MinFeasSDP) to obtain a solution $X_{\text {infeas }}$. Then set $\delta=\gamma\left\|\mathcal{A}_{V}\left(X_{\text {infeas }}\right)-b\right\|_{2}$ for some $\gamma \geq 1$. This choice guarantees that (MinObjSDP) is feasible. In our numerics, we find $\gamma=1.1$ works well. ${ }^{2}$

5.4. Bounds on Suboptimality, Infeasibility, and Distance to the Solution Set. Suppose we solve either (MinObjSDP) or (MinFeasSDP) to obtain a primal estimate $X=X_{\mathrm{obj}}$ or $X=X_{\text {infeas }}$. How can we estimate the suboptimality, infeasibility, and distance of $X$ to the solution set of $(\mathrm{P})$ ?

\footnotetext{
2 It is possible to directly set the value $\delta$ without solving (MinFeasSDP) using the bounds from Theorem D.1 in Appendix D when additional information or computation budget is available. However, evaluating the bounds (which can be potentially loose) might be as hard as solving (MinFeasSDP).
} 
The first two metrics are straightforward to compute. We can bound the suboptimality by $\epsilon_{p}(X) \leq \operatorname{tr}(C X)-b^{\top} y$. We can compute the infeasibility as $\delta_{p}(X)=$ $\|\mathcal{A} X-b\|_{2}$. In the optimization literature, scaled versions of the suboptimality and infeasibility called KKT residuals [71, 47, 77] are generally used as stopping criteria.

The distance to the solution requires additional assumptions, such as surjectivity of the restricted constraint map $\mathcal{A}_{V}$. With these assumptions, Lemma 4.4 yields a computable (but possibly loose) bound. We refer the interested reader to Appendix D.

6. Computational Aspects of the Dual SDP (D). The previous two subsections showed how to efficiently recover an approximate primal solution from an approximate dual solution. We now discuss how to (approximately) solve the dual SDP (D) with optimal storage and with a low per-iteration computational cost. Together, the (storage-optimal) dual solver and (storage-optimal) primal recovery compose a new algorithm for solving regular SDPs with optimal storage.

6.1. Exact Penalty Formulation. It will be useful to introduce an unconstrained version of the dual SDP (D), parametrized by a real positive number $\alpha$, which we call the penalized dual SDP:

$$
\text { maximize } \quad b^{\top} y+\alpha \min \left\{\lambda_{\min }\left(C-\mathcal{A}^{\top} y\right), 0\right\} .
$$

That is, we penalize vectors $y$ that violate the dual constraint $C-\mathcal{A}^{\top} y \succeq 0$.

Problem (6.1) is an exact penalty formulation for the dual SDP (D). Indeed, the following lemma shows that the solution of Problem (6.1) and the solution set of the dual SDP (D) are the same when $\alpha$ is large enough. The proof is based on [61, Theorem 7.21].

Lemma 6.1. Instate the assumptions in Subsection 1.1. If $b \neq 0$ and $\alpha>\left\|X_{\star}\right\|_{*}$, then the penalized dual SDP (6.1) and the dual SDP (D) have the same solution $y_{\star}$.

Proof of Lemma 6.1. We first note that the dual solution $y_{\star}$ is the only solution to $\min _{\lambda_{\max }\left(\mathcal{A}^{\top} y-C\right) \leq 0}-b^{\top} y$. Using [61, Theorem 7.21], we know that the penalty form (6.1) has $y_{\star}$ as its only solution as long as $\alpha>\alpha_{0}$ for any $\alpha_{0} \geq 0$ satisfying the KKT condition:

$$
b \in \alpha_{0} \mathcal{A}\left(\partial\left(\lambda_{\max }\left(-Z\left(y_{\star}\right)\right)\right)\right), \quad \text { and } \quad \alpha_{0} \lambda_{\max }\left(-Z\left(y_{\star}\right)\right)=0 .
$$

This is the case by noting $X_{\star} \in \operatorname{tr}\left(X_{\star}\right) \partial\left(\lambda_{\max }\left(-Z\left(y_{\star}\right)\right), \operatorname{tr}\left(X_{\star}\right) \lambda_{\max }\left(-Z\left(y_{\star}\right)\right)=0\right.$, and $\mathcal{A}\left(X_{\star}\right)=b$. Hence we can choose $\alpha_{0}=\operatorname{tr}\left(X_{\star}\right)$.

Thus, as long as we know an upper bound on the nuclear norm of the primal solution, then we can solve Problem (6.1) to find the dual optimal solution $y_{\star}$. It is often easy to find a bound on $\left\|X_{\star}\right\|_{*}$ in the following two situations:

1. Nuclear norm objective. Suppose the objective in $(\mathrm{P})$ is $\|X\|_{*}=\operatorname{tr}(X)$. Problems using this objective include matrix completion [19], phase retrieval [21], and covariance estimation [23]. In these settings, it is generally easy to find a feasible solution or to bound the objective via a spectral method. (See [39] for matrix completion and [18] for phase retrieval.)

2. Constant trace constraints. Suppose the constraint $\mathcal{A} X=b$ enforces $\operatorname{tr}(X)=$ $\beta$ for some constant $\beta$. Problems with this constraint include Max-Cut [32], Community Detection [48], and PhaseCut in [69]. Then any $\alpha>\beta$ serves as an upper bound. In the Powerflow $[7,46]$ problems, we have constraints: $X_{i i} \leq \beta_{i}, \forall i$. Then any $\alpha>\sum_{i=1}^{n} \beta_{i}$ serves as an upper bound. (The Powerflow problem does not directly fit into our standard form (P), but a small modification of our framework can handle the problem.) 


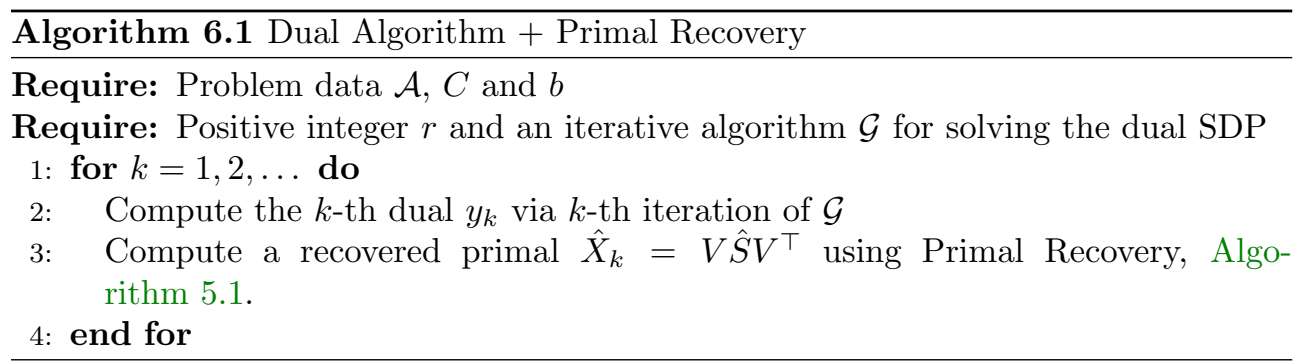

When no such bound is available, we may search over $\alpha$ numerically. For example, solve Problem (6.1) for $\alpha=2,4,8, \ldots, 2^{d}$ for some integer $d$ (perhaps, in parallel, simultaneously). Since any feasible $y$ for the dual SDP (D) may be used to recover the primal, using (MinFeasSDP) and (MinFeasSDP), we can use any approximate solution of the penalized dual SDP, Problem (6.1), for any $\alpha$, as long as it is feasible for the dual SDP.

Alternatively, the method in [59] which solves (D) directly can also be utilized if a strictly dual feasible point is known. For example, $C \succ 0$ and $0 \in \mathbf{R}^{m}$ is a strictly dual feasible point.

6.2. Computational Cost and Convergence Rate for Primal Approximation. Suppose we have an iterative algorithm $\mathcal{G}$ to solve the dual problem. Denote by $y_{k}$ the $k$ th iterate of $\mathcal{G}$. Each dual iterate $y_{k}$ generates a corresponding primal iterate using either (MinFeasSDP) or (MinFeasSDP). We summarize this approach to solving the primal SDP in Algorithm 6.1.

The primal iterates $X_{k}$ generated by Algorithm 6.1 converge to a solution of the primal SDP (P) by our theory. ${ }^{3}$ However, it would be computational folly to recover the primal at every iteration: the primal recovery problem is much more computationally challenging than a single iteration of most methods for solving the dual. Hence, to determine when (or how often) to recover the primal iterate from the dual, we would like to understand how quickly the recovered primal iterates converge to the solution of the primal problem.

To simplify exposition as we discuss algorithms for solving the dual, we reformulate the penalized dual SDP as a convex minimization problem,

$$
\text { minimize } g_{\alpha}(y):=-b^{\top} y+\alpha \max \left\{\lambda_{\max }\left(-C+\mathcal{A}^{\top} y\right), 0\right\},
$$

which has the same solution set as the penalized dual SDP (6.1).

We focus on the convergence of suboptimality and infeasibility, as these two quantities are easier to measure than distance to the solution set. Recall from Table 3 that

$$
\epsilon \text {-optimal dual feasible } y \underset{\text { or MinFeasSDP }}{\stackrel{\text { MinObjSDP }}{\longrightarrow}}(\mathcal{O}(\sqrt{\epsilon}), \mathcal{O}(\sqrt{\epsilon})) \text {-primal solution } X
$$

if $\kappa=\mathcal{O}(1)$. Thus the convergence rate of the primal sequence depends strongly on the convergence rate of the algorithm we use to solve the penalized dual SDP.

\footnotetext{
${ }^{3}$ Iterative algorithms for solving the dual SDP (D) may not give a feasible point $y$. If a strictly feasible point is available, we can use the method of Lemma E.1 or Lemma E.2 in the appendix to obtain a sequence of feasible points from a sequence of (possibly infeasible) iterates without affecting the convergence rate. Alternatively, our theory can be extended to handle the infeasible case; we omit this analysis for simplicity.
} 
6.2.1. Subgradient Methods, Storage Cost, and Per-Iteration Time Cost. We focus on subgradient-type methods for solving the penalized dual SDP (6.1), because the objective $g_{\alpha}$ is nonsmooth but has an efficiently computable subgradient. Any subgradient method follows a recurrence of the form

$$
y_{0} \in \mathbf{R}^{m} \text { and } y_{k+1}=y_{k}-\eta_{k} g_{k},
$$

where $g_{k}$ is a subgradient of $g_{\alpha}$ at $y_{k}$ and $\eta_{k} \geq 0$ is the step size. Subgradienttype methods differ in the methods for choosing the step size $\eta_{k}$ and in their use of parallelism. However, they are all easy to run for our problem because it is easy to compute a subgradient of the dual objective with penalty $g_{\alpha}$ :

Lemma 6.2. Let $Z=C-\mathcal{A}^{\top} y$. The subdifferential of the function $g_{\alpha}$ is

$$
\partial g_{\alpha}(y)= \begin{cases}-b+\operatorname{conv}\left\{\alpha \mathcal{A}\left(v v^{\top}\right) \mid Z v=\lambda_{\min }(Z) v\right\}, & \lambda_{\min }(Z)<0 \\ -b, & \lambda_{\min }(Z)>0 \\ -b+\beta \operatorname{conv}\left\{\alpha \mathcal{A}\left(v v^{\top}\right) \mid Z v=\lambda_{\min }(Z) v, \beta \in[0,1]\right\}, & \lambda_{\min }(Z)=0\end{cases}
$$

This result follows directly via standard subdifferential calculus from the subdifferential of the maximum eigenvalue $\lambda_{\max }(\cdot)$. Thus our storage cost is simply $\mathcal{O}(m+n)$ where $m$ is due to storing the decision variable $y$ and the gradient $g_{k}$, and $n$ is due to the intermediate eigenvector $v \in \mathbf{R}^{n}$. The main computational cost in computing a subgradient of the objective in (6.2) is computing the smallest eigenvalue $\lambda_{\min }\left(C-\mathcal{A}^{\top} y\right)$ and the corresponding eigenvector $v$ of the matrix $C-\mathcal{A}^{\top} y$. Since $C-\mathcal{A}^{\top} y$ can be efficiently applied to vectors (using the data access oracles (1.4)), we can compute this eigenpair efficiently using the randomized Lanczos method [40].

6.2.2. Convergence Rate of the Dual and Primal. The best available subgradient method [38] has convergence rate $\mathcal{O}(1 / \epsilon)$ when the quadratic growth condition is satisfied. (This result does not seem to appear in the literature for SDP; however, it is a simple consequence of [38, Table 1] together with the quadratic growth condition proved in Lemma 3.1.) Thus, our primal recovery algorithm has convergence rate $\mathcal{O}(1 / \sqrt{\epsilon})$, using the relation between dual convergence and primal convergence in (6.3). Unfortunately, the algorithm in [38] involves many unknown constants. In practice, we recommend using dual solvers that require less tuning such as AcceleGrad [44] which is the one we used in Section 7.

7. Numerical Experiments. In this section, we give a numerical demonstration of our approach to solving $(\mathrm{P})$ via approximate complementarity. We first show that Algorithm 5.1 (Primal Recovery) recovers an approximate primal given an approximate dual solution. Next, we show that Algorithm 6.1 with primal recovery achieves reasonable accuracy $\left(10^{-1} \sim 10^{-2}\right)$ for extremely large scale problems, e.g., $10^{5} \times 10^{5}$, with substantially lower storage requirements compared to other SDP solvers.

We test our methods on the Max-Cut and Matrix Completion SDPs, defined in Table 5. For Max-Cut, $L$ is the Laplacian of a given graph. For Matrix Completion, $\Omega$ is the set of indices of the observed entries of the underlying matrix $\bar{X} \in \mathbf{R}^{n_{1} \times n_{2}}$. We use the dual penalty form (6.2) which defines defines $g_{\alpha}$ to measure both dual suboptimality and infeasibility. We set $\alpha=1.1 n$ for Max-Cut and $2.2 \times\|\bar{X}\|_{*}$ for matrix completion throughout our experiments.

7.1. Primal Recovery. Our first experiment confirms numerically that Algorithm 5.1 (Primal Recovery) recovers an approximate primal from an approximate 
Table 5: Problems for numerics

\begin{tabular}{|ll|ll|}
\hline \multicolumn{2}{|c|}{ Max-Cut } & \multicolumn{2}{c|}{ Matrix Completion } \\
\hline minimize & $\operatorname{tr}(-L X)$ & minimize & $\operatorname{tr}\left(W_{1}\right)+\operatorname{tr}\left(W_{2}\right)$ \\
subject to & $\operatorname{diag}(X)=\mathbf{1}$ & subject to & $X_{i j}=\bar{X}_{i j},(i, j) \in \Omega$ \\
& $X \succeq 0$ & & {$\left[\begin{array}{cc}W_{1} & X \\
X^{\top} & W_{2}\end{array}\right] \succeq 0$} \\
\hline
\end{tabular}

dual solution, validating our theoretical results. As an example, we present results for the Max-Cut SDP using a Laplacian $L \in \mathbf{R}^{800 \times 800}$ from the G1 dataset of [2]. Results for matrix completion and for other Max-Cut problems are similar; we present the corresponding experiment for matrix completion in Appendix F.1. To evaluate our method, we compare the recovered primal with the primal dual solution $X_{\star}, y_{\star}$ obtained with Sedumi, an interior point solver [63]. Empirically, the rank of the primal solution $r_{\star}=13$.

To obtain approximate dual solutions $y$, we perturb the true dual solution $y_{\star}$ to generate

$$
y=y_{\star}+\varepsilon s\left\|y_{\star}\right\|_{2},
$$

where $\varepsilon$ is the noise level, which we vary from 1 to $10^{-5}$, and $s$ is a uniformly random vector on the unit sphere in $\mathbf{R}^{m}$. For each perturbed dual $y$ and for each rank estimate $r \in\left\{r_{\star}, 3 r_{\star}\right\}$, we first solve (MinFeasSDP) to obtain a solution $X_{\text {infeas }}$, and then solve (MinObjSDP) with $\delta=1.1\left\|\mathcal{A} X_{\text {infeas }}-b\right\|_{2}$. We measure the suboptimality of the perturbed dual using the relative suboptimality $\frac{\left|p^{\star}+g_{\alpha}(y)\right|}{\left|p^{\star}\right|}$. We measure the distance of the recovered primal to the solution in three ways: relative suboptimality $\left|\operatorname{tr}(C X)-p_{\star}\right| / p_{\star}$, relative infeasibility $\|\mathcal{A} X-b\| /\|b\|$, and relative distance to the solution set $\left\|X-X_{\star}\right\|_{\mathrm{F}} /\left\|X_{\star}\right\|_{\mathrm{F}}$.

Figure 1 shows distance of the recovered primal to the solution. The blue dots show the primal recovered using $r=r_{\star}$, while the red dots show the primal recovered using $r=3 r_{\star}$. The blue and red curves are fit to the dots of the same color to provide a visual guide. The red line $\left(r=3 r_{\star}\right)$ generally lies below the blue line $\left(r=r_{\star}\right)$, which confirms that larger ranks produce more accurate primal reconstructions.

These plots show that the recovered primal approaches the true primal solution as the dual suboptimality approaches zero, as expected from our theory. ${ }^{4}$ From Table 3, recall that we expect the primal solution recovered from an $\epsilon$ suboptimal dual solution to converge to the true primal solution as $\mathcal{O}(\sqrt{\epsilon})$ with respect to all three measures. The plots confirm this scaling for distance to solution and infeasibility, while suboptimality decays even faster than predicted by our theory. By construction, the primal suboptimality of (MinObjSDP) is smaller than that of (MinFeasSDP); however, the plots measure primal suboptimality by its absolute value. The kink in the curves desribing primal suboptimality for (MinObjSDP) separates suboptimal primal solutions (to the left) from superoptimal solutions (to the right). Finally, notice that $3 r_{\star}=39$ is close to the Barvinok-Pataki bound. Interestingly, (MinFeasSDP) still performs better with this large feasible set $\left(r=3 r_{\star}\right)$ than with a smaller one $\left(r=r_{\star}\right)$, although our theory does not apply.

\footnotetext{
${ }^{4}$ To be precise, the theory we present above in Theorem 4.1 and Theorem 4.6 requires the approximate dual solution to be feasible, while $y$ may be infeasible in our experiments. An extension of our results can show similar bounds when $y$ is infeasible but $g_{\alpha}(y)$ is close to $-d_{\star}$.
} 

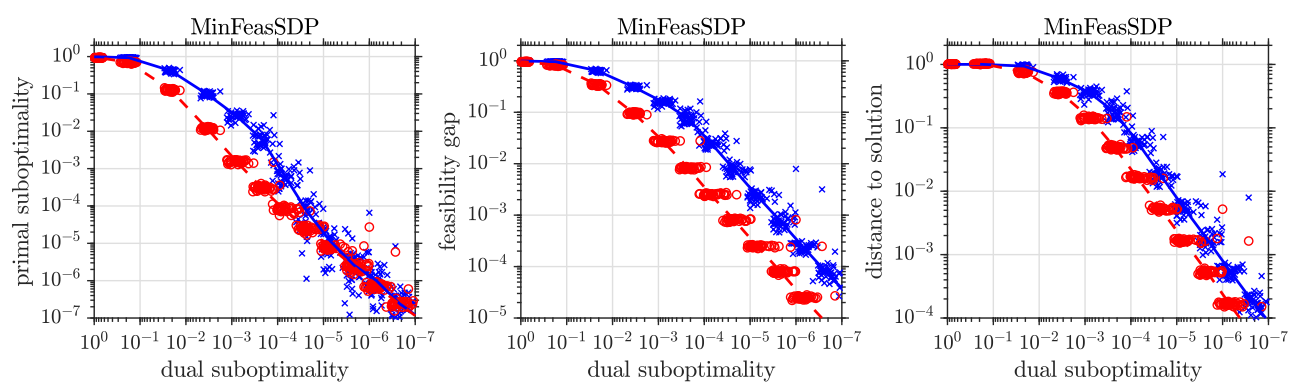

(a) (MinFeasSDP)
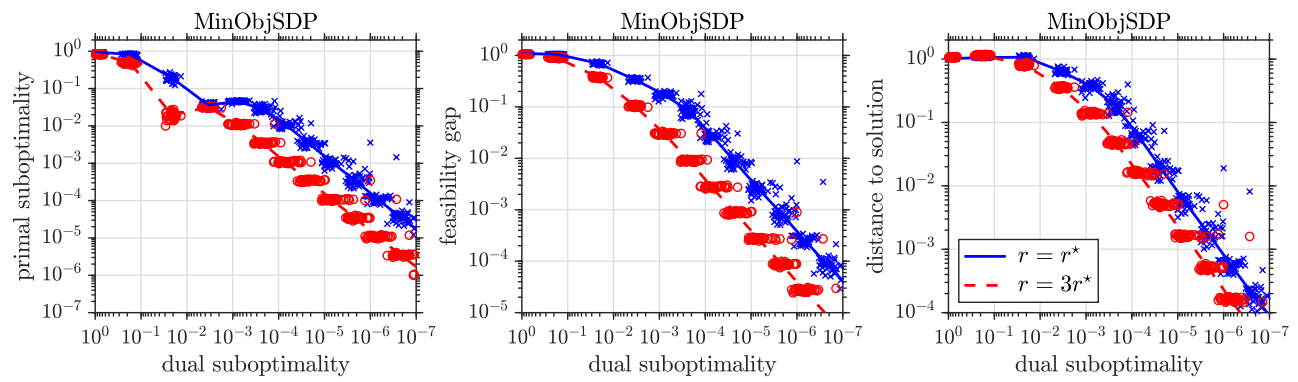

(b) (MinObjSDP)

Fig. 1: The plots shows the primal recovery performance of (MinFeasSDP) (upper) and (MinObjSDP) (lower) in terms of (relative) primal suboptimality $\mid \operatorname{tr}(C X)-$ $p_{\star} \mid / p_{\star}$, (relative) infeasibility gap $\|\mathcal{A} X-b\| /\|b\|$, and (relative) distance to solution $\left\|X-X_{\star}\right\|_{\mathrm{F}} /\left\|X_{\star}\right\|_{\mathrm{F}}$. The horizontal axis is (relative) dual suboptimality $\frac{\left|p^{\star}+g_{\alpha}(y)\right|}{\left|p^{\star}\right|}$. The blue dots corresponds to the choice $r=r_{\star}$ and the red dots corresponds to the choice $r=3 r_{\star}$ in Algorithm 6.1.

7.2. Storage efficiency comparing to existing solvers. Experiments in this section show that our method Algorithm 6.1 uses less storage (for high dimensional problems) than existing algorithms. We use AccelGrad [44] as the dual solver. We solve (MinObjSDP) to recover the primal, using $\gamma=1.1$ and several different rank estimates $r$. We compare Algorithm 6.1 against the mature SDP solvers Mosek [50], SDPT3 [67] and Sedumi [63], and the state-of-the-art SDP solver SDPNAL+ [65]. Figure 2 (our method is labeled as CSSDP) shows how the storage required for these methods scales with the side length $n$ of the primal decision variable $X \in \mathbf{S}^{n}$ for MaxCut and matrix completion. Our Max-Cut problems are drawn from Gset and the DIMACS10 group [1]. Our matrix completion problems are simulated. We generate rank 5 random matrices $\bar{X}=U V \in \mathbf{R}^{n_{1} \times n_{2}}$ where $U \in\{ \pm 1\}^{n_{1} \times 5}$ and $V \in\{ \pm 1\}^{5 \times n_{2}}$ are random sign matrices. We vary the dimensions by setting $n=75 \mathrm{c}, \mathrm{m}=50 \mathrm{c}$ and varying $c=1,2,4,8, \ldots$ The $25\left(n_{1}+n_{2}\right) \log \left(n_{1}+n_{2}\right)$ observations are chosen uniformly at random.

As can be seen from the plots, the mature solvers Mosek, SDPT4, and Sedumi exceed the storage limit 16GB for matrix completion when $n>10^{3}$ and for Max-Cut when $n>10^{4}$. SDPNAL+ uses less storage than the mature solvers. However, the 


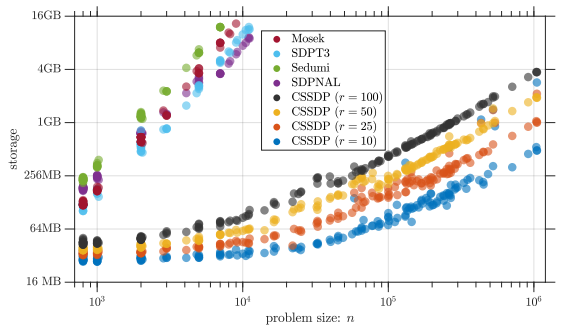

(a) Max-Cut

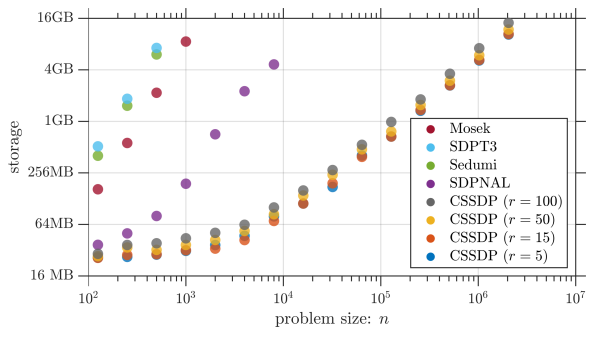

(b) Matrix Completion

Fig. 2: Here we compare our Algorithm 6.1 (shown as CSSDP with different choice of $r$ ), with Mosek, SDPT3, Sedumi, and SDPNAL+. Dots for Mosek, SDPT3, Sedumi, and SDPNAL+ are not shown for large $n$ because they exceed 16GB.

storage still exceeds $16 \mathrm{~GB}$ when $n>10^{4}$ for both problems. In contrast, our method (labeled as CSSDP) scales linearly with the dimension (for any $r$ ), and can solve problems with $n=10^{6}$ on a 16GB laptop.

7.3. Accuracy versus time. In this section, we demonstrate that Algorithm 6.1 can solve large scale problems that are inaccesible to the SDP solvers Mosek, SDPT3, Sedumi, and SDPNAL+ due to storage limit. Since primal recovery is substantially more expensive than a dual iteration (see Subsection 6.2), we recover the primal only at iterations $10,10^{2}, 10^{3}, 10^{4}, 10^{5}, \ldots$ We solve both (MinFeasSDP)) (option 1) and (MinObjSDP)) with $\gamma=1.1$ (option 2) in Algorithm 5.1. These solutions are shown as the solid and dotted lines in Figure 3, respectively. Since we do not know the optimal solution, we track performance using two DIMACS measures of (scaled) infeasibility and suboptimality,

$$
\begin{array}{cl}
\text { relative feasibility gap: } & \frac{\|\mathcal{A}(X)-b\|_{2}}{\|b\|_{2}+1} \\
\text { relative primal-dual gap: } & \frac{\left|\operatorname{tr}(C X)+g_{\alpha}(y)\right|}{|\operatorname{tr}(C X)|+\left|g_{\alpha}(y)\right|+1} .
\end{array}
$$

These measures are commonly used to benchmark SDP solvers [71, 47, 77]. Here $\operatorname{tr}(C X)+g_{\alpha}(y)$ bounds primal suboptimality. It is traditional to use $\operatorname{tr}(C X)-b^{\top} y$; however, here $y$ is not necessarily dual feasible and so this simpler measure does not bound primal suboptimality. Results for the Max-Cut SDP on the smallworld graph in the DIMACS10 group [1], with a decision variable of size $10^{5} \times 10^{5}$, are shown in Figure 3a. Results for a matrix completion problem, simulated as described in Subsection 7.2 with $c=1000$, with decision variable size $\left(n_{1}+n_{2}\right)^{2}=\left(1.25 \times 10^{5}\right)^{2}$ with $n_{1}=75000$ and $n_{2}=50000$, and over $3.6 \times 10^{7}$ many constraints are shown in Figure $3 \mathrm{~b}$.

As can be seen, the proposed method reaches a solution with $10^{-1}$ infeasibility and $10^{-3}$ suboptimality in $10^{4}$ seconds when the rank parameter is large $(r=100$ or $r=$ 250) for Max-Cut and $10^{-1}$ infeasibility and $10^{-3}$ suboptimality in $10^{5}$ seconds when $r=5$ or 15 for matrix completion. These ranks are far smaller than the BarvinokPataki bound. Again, (MinFeasSDP) outperforms (MinObjSDP) and is faster and easier to compute. We plot points according to their (dual) iteration counter; the top 

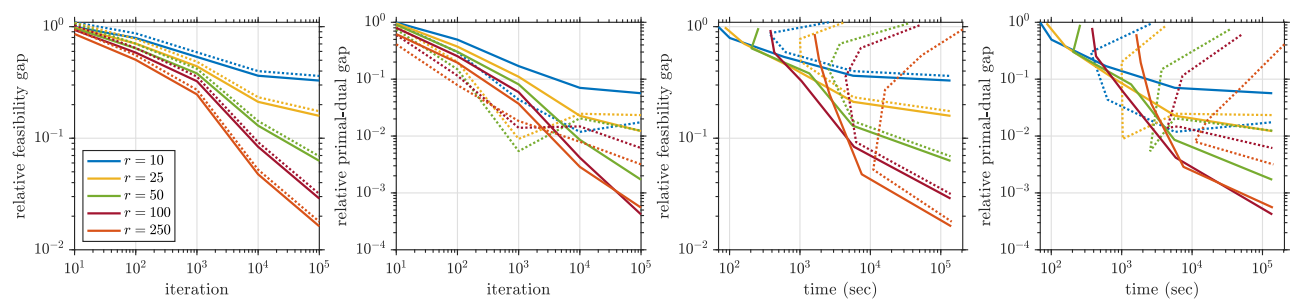

(a) Max-Cut: smallworld
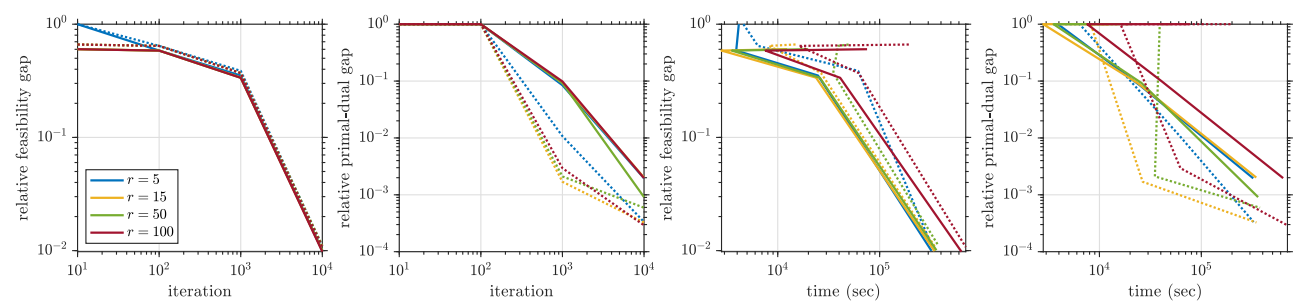

(b) MatrixCompletion: $n_{1}=75000, \quad n_{2}=50000$

Fig. 3: Convergence of Algorithm 6.1 with (MinFeasSDP) as the solid line and (MinObjSDP) as the dotted line. Primal recovery from accurate dual iterates is both more accurate and faster, so primal iterates recovered from early dual iterates can be dominated by those recovered from later iterates.

point on each line corresponds to dual iteration 10. Primal recovery from accurate dual iterates is both more accurate and faster, so primal iterates recovered from early dual iterates can be dominated by those recovered from later iterates.

Additional experiments can be found in Appendix F.

8. Conclusions. This paper presents a new theoretically justified method to recover an approximate solution to a primal SDP from an approximate solution to a dual SDP, using complementarity between the primal and dual optimal solutions. We present two concrete algorithms for primal recovery, which offer guarantees on the suboptimality, infeasibility, and distance to the solution set of the recovered primal under the regular conditions on the SDP, and we demonstrate that this primal recovery method works well in practice.

We use this primal recovery method to develop the first storage-optimal algorithm to solve regular SDP: use any first-order algorithm to solve a penalized version of the dual problem, and recover a primal solution from the dual. This method requires $O(m+n r)$ storage: the storage is linear in the number of constraints $m$ and in the side length $n$ of the SDP variable, when the target rank $r$ of the solution is fixed. These storage requirements improve on the storage requirements that guarantee convergence for nonconvex factored (Burer-Monteiro) methods to solve the SDP, which scale as $O(\sqrt{m} n)$. Furthermore, we show that no method can use less storage without a more restrictive data access model or a more restrictive representation of the solution. We demonstrate numerically that our algorithm is able to solve SDP of practical interest including Max-Cut and Matrix Completion.

The ideas illustrated in this paper can be extended to solve problems with in- 
equality constraints. We leave this extension for future work.

Appendix A. Lemmas for Section 1. To establish Lemma 3.1, we prove a lemma concerning the operator $\mathcal{A}_{V_{\star}}$.

Lemma A.1. Instate the hypothesis of Subsection 1.1. Then $\operatorname{null}\left(\mathcal{A}_{V^{\star}}\right)=\{0\}$.

Proof of Lemma A.1. Suppose by way of contradiction that $\operatorname{ker}\left(\mathcal{A}_{V_{\star}}\right) \neq\{0\}$. Let $S \in \operatorname{ker}\left(\mathcal{A}_{V_{\star}}\right)$, so $\mathcal{A}_{V_{\star}}(S)=0$. Recall $X_{\star}=V_{\star} S_{\star}\left(V_{\star}\right)^{\top}$ for some unique $S_{\star} \succ 0$. Hence for some $\alpha_{0}>0, S_{\star}+\alpha S \succeq 0$ for all $|\alpha| \leq \alpha_{0}$. Now pick any $\alpha$ with $|\alpha| \leq \alpha_{0}$ to see

$$
\mathcal{A}\left(X_{\alpha}\right)=\mathcal{A}_{V_{\star}}\left(S_{\star}+\alpha S\right)=\mathcal{A}_{V_{\star}}\left(S_{\star}\right)+0=b .
$$

This shows $X_{\alpha}$ is feasible for all $|\alpha| \leq \alpha_{0}$. But we can always find some $|\alpha| \leq \alpha_{0}, \alpha \neq$ 0 , so that $\operatorname{tr}\left(C X_{\alpha}\right)=p_{\star}+\alpha \operatorname{tr}\left(C V_{\star}\left(S V_{\star}\right)^{\top}\right) \leq p_{\star}$. This contradicts the assumption that $X_{\star}$ is unique. Hence we must have $\operatorname{null}\left(\mathcal{A}_{V_{\star}}\right)=\{0\}$.

Proof of Lemma 3.1. Consider the linear operator $\mathcal{D}$ defined in Lemma 3.1. An argument similar to the proof of Lemma A.1 shows $\operatorname{ker}(\mathcal{D})=\{0\}$ (see Lemma A.2 below for more details). Hence

$$
\left\|(Z(y), y)-\left(Z\left(y_{\star}\right), y_{\star}\right)\right\| \leq \frac{1}{\sigma_{\min }(\mathcal{D})}\left\|\mathcal{D}\left(Z(y)-Z\left(y_{\star}\right), y-y_{\star}\right)\right\| .
$$

By utilizing Lemma 4.3 with $X=Z(y)$ and $Z=X_{\star}$ and note $\epsilon=\operatorname{tr}\left(Z(y) X_{\star}\right)=$ $\epsilon_{d}(y)=b^{\top} y_{\star}-b^{\top} y$ (from strong duality), we see that

$$
\left\|Z(y)-\left(U^{\star}\right)\left(U^{\star}\right)^{\top} Z(y)\left(U^{\star}\right)\left(U^{\star}\right)^{\top}\right\|_{\mathrm{F}} \leq \frac{\epsilon}{\lambda_{\min >0}\left(X_{\star}\right)}+\sqrt{\frac{2 \epsilon}{\lambda_{\min >0}\left(X_{\star}\right)}\|Z(y)\|_{\mathrm{op}}} .
$$

We also have

$$
\mathcal{D}\left(Z(y)-Z\left(y_{\star}\right), y-y_{\star}\right)=(\mathcal{D} Z(y), 0)=Z(y)-\left(U^{\star}\right)\left(U^{\star}\right)^{\top} Z(y)\left(U^{\star}\right)\left(U^{\star}\right)^{\top} .
$$

Combining the above pieces, we get the results in Lemma 3.1.

Lemma A.2. Instate the hypothesis of Subsection 1.1. Then $\operatorname{null}(\mathcal{D})=\{0\}$.

Proof. Suppose $\mathcal{D} \neq\{0\}$, then there is some $\hat{Z}$ and $\hat{y}$ such that

$$
\mathcal{D}(\hat{Z}, \hat{y})=0 \Longrightarrow \hat{Z}=-\mathcal{A}^{\top} \hat{y} \quad \text { and } \quad \hat{Z}=\left(U_{\star} U_{\star}^{\top}\right) \hat{Z}\left(U_{\star} U_{\star}^{\top}\right)
$$

Then we claim $y_{\gamma}=y_{\star}+\gamma \hat{y}$ is also an solution to the dual SDP (D), which violates the unique assumption. Indeed, $Z\left(y_{\gamma}\right)$ satisfies

$$
\begin{aligned}
Z\left(y_{\gamma}\right) & =C-\mathcal{A}^{\top}\left(y_{\gamma}\right) \\
& =Z\left(y_{\star}\right)-\gamma \mathcal{A}^{\top} \hat{y} \\
& \stackrel{(a)}{=} Z\left(y_{\star}\right)+\gamma \hat{Z} \\
& \stackrel{(b)}{=} U_{\star}\left(U_{\star}^{\top} Z\left(y_{\star}\right) U_{\star}+\gamma U_{\star}^{\top} \hat{Z} U_{\star}\right) U_{\star}^{\top}
\end{aligned}
$$

Here we use the $\mathcal{D}(\hat{Z}, \hat{y})=0$ in step $(a)$ and $(b)$. Because of strict complementarity, $\operatorname{range}\left(U_{\star}\right)=\operatorname{range}\left(Z\left(y_{\star}\right)\right)$ and hence $U_{\star}^{\top} Z\left(y_{\star}\right) U_{\star} \succ 0$. Hence there is some constant $c>0$ such that for all $|\gamma|<c, Z\left(y_{\gamma}\right)$ is still feasible for the dual SDP (D). But the objective $b^{\top} y_{\star}+\gamma b^{\top} \hat{y}$ then can be larger to $b^{\top} y_{\star}$ for all $|\gamma|<c$ and $\gamma$ matching the sign of $b^{\top} \hat{y}$, and equal to $b^{\top} y_{\star}$ for all $|\gamma|<c$ if $b^{\top} \hat{y}=0$.

Appendix B. Lemmas from Section 4. We first prove Lemma 4.3 concerning the distance to subspaces and the inner product. 
Proof of Lemma 4.3. Complete $V$ to form a basis $W=[U V]$ for $\mathbf{R}^{n}$, where $U=\left[v_{r+1}, \ldots, v_{n}\right] \in \mathbf{R}^{n \times(n-r)}$ and where $v_{i}$ is the eigenvector of $Z$ associated with the $i$-th smallest eigenvalue.

Rotating into the coordinate system formed by $W=[V ; U]$, let us compare $X$ and its projection into the space spanned by $V, P_{V}(X):=V V^{\top} X V V^{\top}$,

$$
W^{\top} X W^{=}=\left[\begin{array}{cc}
U^{\top} X U & U^{\top} X V \\
V^{\top} X U & V^{\top} X V
\end{array}\right], \quad \text { and } \quad W^{\top} P_{V}(X) W=\left[\begin{array}{cc}
0 & 0 \\
0 & V^{\top} X V
\end{array}\right] .
$$

Let $X_{1}=U^{\top} X U, B=U^{\top} X V$ and $X_{2}=V^{\top} X V$. Using the unitary invariance of $\|\cdot\|_{\mathrm{F}}$, we have $P_{V^{\perp}}(X):=X-V V^{\top} X V V^{\top}$ satisfying

$$
\left\|P_{V^{\perp}}(X)\right\|_{\mathrm{F}}=\left\|W^{\top} X W-W^{\top} V V^{\top} X V V^{\top} W\right\|_{\mathrm{F}}=\left\|\left[\begin{array}{cc}
X_{1} & B \\
B & 0
\end{array}\right]\right\|_{\mathrm{F}} .
$$

A similar equality holds for $\|\cdot\|_{*}$. Thus we need only bound the terms $X_{1}$ and $B$. Applying Lemma B.1 to $W X W^{\top}=\left[\begin{array}{cc}X_{1} & B \\ B^{\top} & X_{2}\end{array}\right]$, we have

$$
\left\|X_{2}\right\|_{\mathrm{op}} \operatorname{tr}\left(X_{1}\right) \geq\left\|B B^{\top}\right\|_{*} .
$$

Since all the vectors in $U$ have corresponding eigenvalues at least as large as the threshold $T=\lambda_{n-r}(Z)>0$, and $Z \succeq 0$ by assumption, we have

$$
\epsilon=\operatorname{tr}(Z X)=\sum_{i=1}^{n} \lambda_{n-i+1}(Z) v_{i}^{\top} X v_{i} \geq \lambda_{n-r}(Z) \sum_{i=r+1}^{n} v_{i}^{\top} X v_{i}
$$

This inequality allows us to bound $\left\|X_{1}\right\|_{\mathrm{F}}$ as

$$
\frac{\epsilon}{T} \geq \sum_{i=r+1}^{n} v_{i}^{\top} X v_{i}=\operatorname{tr}\left(U X U^{\top}\right)=\operatorname{tr}\left(X_{1}\right)=\left\|X_{1}\right\|_{*} \geq\left\|X_{1}\right\|_{\mathrm{F}},
$$

where we recall $X_{1} \succeq 0$ to obtain the second to last equality. Combining (B.2), (B.4), and $\left\|X_{2}\right\|_{\mathrm{op}} \leq\|X\|_{\mathrm{op}}$, we have

$$
\left\|B B^{\top}\right\|_{*} \leq \frac{\epsilon}{T}\left\|X_{2}\right\|_{\mathrm{op}} \leq \frac{\epsilon}{T}\|X\|_{\mathrm{op}} .
$$

Basic linear algebra shows

$$
\left\|\left[\begin{array}{cc}
0 & B \\
B^{\top} & 0
\end{array}\right]\right\|_{\mathrm{F}}^{2}=\operatorname{tr}\left(\left[\begin{array}{cc}
B B^{\top} & 0 \\
0 & B^{\top} B
\end{array}\right]\right) \leq 2 \operatorname{tr}\left(B B^{\top}\right)=2\left\|B B^{\top}\right\|_{*} .
$$

Combining pieces, we bound the error in the Frobenius norm:

$$
\begin{aligned}
\left\|X-V V^{\top} X V V^{\top}\right\|_{\mathrm{F}} & \stackrel{(a)}{\leq}\left\|X_{1}\right\|_{\mathrm{F}}+\left\|\left[\begin{array}{cc}
0 & B \\
B^{\top} & 0
\end{array}\right]\right\|_{\mathrm{F}} \stackrel{(b)}{\leq} \frac{\epsilon}{T}+\sqrt{2\left\|B B^{\top}\right\|_{*}} \\
& \stackrel{(c)}{\leq} \frac{\epsilon}{T}+\sqrt{\frac{2 \epsilon}{T}\|X\|_{\mathrm{op}}},
\end{aligned}
$$


where step (a) uses (B.1) and the triangle inequality; step (b) uses (B.2) and (B.6); and step $(c)$ uses (B.5). Similarly, we may bound the error in the nuclear norm:

$$
\begin{aligned}
\left\|X_{\star}-V V^{\top} X_{\star} V V^{\top}\right\|_{*} & \stackrel{(a)}{\leq}\left\|X_{1}\right\|_{*}+\left\|\left[\begin{array}{cc}
0 & B \\
B^{\top} & 0
\end{array}\right]\right\|_{*} \stackrel{(b)}{\leq} \operatorname{tr}\left(X_{1}\right)+\sqrt{2 r}\left\|\left[\begin{array}{cc}
0 & B \\
B^{\top} & 0
\end{array}\right]\right\|_{\mathrm{F}} \\
& \stackrel{(c)}{\leq} \frac{\epsilon}{T}+2 \sqrt{\frac{r \epsilon}{T}\left\|X_{\star}\right\|_{\mathrm{op}}}
\end{aligned}
$$

Step (a) follows step $(a)$ in (B.7). Step $(b)$ uses the fact that $\left[\begin{array}{cc}0 & B \\ B^{\top} & 0\end{array}\right]$ has rank at most $2 r$. Step $(c)$ follows the step $(b)$ and $(c)$ in the inequality (B.7).

Lemma B.1. Suppose $Y=\left[\begin{array}{cc}A & B \\ B^{\top} & D\end{array}\right] \succeq 0$. Then $\|A\|_{\mathrm{op}} \operatorname{tr}(D) \geq\left\|B B^{\top}\right\|_{*}$.

Proof. For any $\epsilon>0$, denote $A_{\epsilon}=A+\varepsilon I$ and $Y_{\epsilon}=\left[\begin{array}{cc}A_{\epsilon} & B \\ B^{\top} & D\end{array}\right]$. We know $Y_{\epsilon}$ is psd, as is its Schur complement $D-B^{\top} A_{\epsilon}^{-1} B \succeq 0$ with $\operatorname{trace} \operatorname{tr}(D)-\operatorname{tr}\left(A_{\epsilon}^{-1} B B^{\top}\right) \geq$ 0 . Von Neumann's trace inequality [49] for $A_{\epsilon}, B B^{\top} \succeq 0$ shows $\operatorname{tr}\left(A_{\epsilon}^{-1} B B^{\top}\right) \geq$ $\frac{1}{\left\|A_{\epsilon}\right\|_{\mathrm{op}}}\left\|B B^{\top}\right\|_{*}$. Use this with $\operatorname{tr}(D)-\operatorname{tr}\left(A_{\epsilon}^{-1} B B^{\top}\right) \geq 0$ to see $\operatorname{tr}(D) \geq \frac{1}{\left\|A_{\epsilon}\right\|_{\mathrm{op}}}\left\|B B^{\top}\right\|_{*}$. Multiply by $\left\|A_{\epsilon}\right\|_{\text {op }}$ and let $\varepsilon \rightarrow 0$ to complete the proof.

Appendix C. Chambolle-Pock for MinObjSDP. Here we state how to use Chambollo-Pock to solve (MinObjSDP):

$$
\begin{array}{ll}
\text { minimize } & \operatorname{tr}\left(C_{V} S\right) \\
\text { subject to } & \left\|\mathcal{A}_{V}(S)-b\right\| \leq \delta \quad \text { and } \quad S \succeq 0 .
\end{array}
$$

In Chambollo-Pock, we have iterates $S_{k} \in \mathbf{S}^{r}, \bar{S}_{k} \in \mathbf{S}^{r}$ and $y_{k} \in \mathbf{R}^{m}$. Denote the projection to the $\delta$-radius ball $\mathbf{B}_{\delta}\left\{y \in \mathbf{R}^{m} \mid\|y\|_{2} \leq \delta\right\}$ as $P_{\mathbf{B}_{\delta}}$ and the projection to $\mathbf{S}_{+}^{r}$ as $P_{\mathbf{S}_{+}^{r}}$. We choose $\tau, \sigma$, and $\theta>0$, and start at $S_{0} \in \mathbf{S}_{+}$. The iteration scheme is as follows:

$$
\begin{aligned}
& y_{k+1}=y_{k}+\sigma\left(\mathcal{A}_{V}\left(\bar{S}_{k}\right)-b\right)-\sigma P_{\mathbf{B}_{\delta}}\left(\frac{1}{\sigma} y_{k}+\mathcal{A}_{V} \bar{S}_{k}-b\right), \\
& S_{k+1}=P_{\mathbf{S}_{+}^{r}}\left(S_{k}-\tau \mathcal{A}_{V}^{\top} y_{k+1}-\tau C_{V}\right), \\
& \bar{S}_{k+1}=S_{k+1}+\theta\left(S^{k+1}-S^{k}\right) .
\end{aligned}
$$

We can compute $C_{V}$ in $r^{2} L_{C}$ time before the iteration scheme and then store it using $r^{2}$ storage. Each iteration only requires one call to each $\mathcal{A}_{V}, \mathcal{A}_{V}^{\top}, P_{\mathbf{B}_{\delta}}$, and $P_{\mathbf{s}_{+}^{r}}$, and a constant number of addition in $\mathbf{R}^{m}$ and $\mathbf{S}_{+}^{r}$, the per iteration flop counts is $\mathcal{O}\left(r^{2} L_{\mathcal{A}}+r L_{\mathcal{A}^{\top}}+m+r^{2} n\right)$. We need to store residues $\mathcal{A}_{V}(S)-b$, one dual iterate of size $m$, two primal iterates of size $r^{2}$ and a few intermediate quantities such as $\mathcal{A}_{V}^{\top} y_{k+1}$ and $\frac{1}{\sigma} y_{k}+\mathcal{A}_{V} \bar{S}_{k}-b$, which requires $\mathcal{O}\left(r^{2}+m\right)$ storage. Hence the method is indeed storage-optimal.

Appendix D. Computable bounds of the distance to solution. We described a way of computing the distance to the solution here, given a bound on $\left\|X_{\star}\right\|_{\mathrm{op}}$ and $\sigma_{\max }(\mathcal{A})$. We note the assumptions here are weaker.

Theorem D.1 (Computable Bounds). Suppose (P) and (D) admit solutions and satisfy strong duality, Equation (1.1). Let $y \in \mathbf{R}^{m}$ be a dual feasible point with 
suboptimality $\epsilon=\epsilon_{d}(y)=b^{\top} y_{\star}-b^{\top} y$. For a positive integer $r$, form the orthonormal matrix $V \in \mathbf{R}^{n \times r}$, as in Algorithm 5.1, and compute the threshold $T=\lambda_{n-r}(Z(y))$.

If $\sigma_{\min }\left(\mathcal{A}_{V}\right)>0$ and $T>0$ and $\left\|X_{\star}\right\|_{\mathrm{op}} \leq B$ for some solution $X_{\star}$ to $(\mathrm{P})$, then

$$
\left\|X_{\text {infeas }}-X_{\star}\right\|_{F} \leq\left(1+\frac{\sigma_{\max }(\mathcal{A})}{\sigma_{\min }\left(\mathcal{A}_{V}\right)}\right)\left(\frac{\epsilon}{T}+\sqrt{2 \frac{\epsilon}{T} B}\right) .
$$

Moreover, any solution $\tilde{S}$ of (MinObjSDP) with infeasibility parameter

$$
\delta \geq \delta_{0}:=\sigma_{\max }(\mathcal{A})\left(\frac{\epsilon}{T}+2 \sqrt{\frac{2 \epsilon B}{T}}\right)
$$

leads to an $\left(\epsilon_{0}, \delta\right)$-solution $X_{o b j}$ for the primal $S D P(\mathrm{P})$ with

$$
\epsilon_{0}=\min \left\{\|C\|_{F}\left(\frac{\epsilon}{T}+\sqrt{2 \frac{\epsilon}{T} B}\right),\|C\|_{\mathrm{op}}\left(\frac{\epsilon}{T}+\sqrt{2 \frac{r \epsilon}{T} B}\right)\right\} .
$$

Proof. The inequality (D.1) is a direct application of Lemma 4.2 and Lemma 4.4. The bound on $\delta_{0}$ and $\epsilon_{0}$ follows the same proof as in Theorem 4.6.

Since $\left\|X_{\star}\right\|_{\text {op }} \in\left[\frac{1}{r_{\star}}\left\|X_{\star}\right\|_{*},\left\|X_{\star}\right\|_{*}\right]$, we might use $\left\|X_{\star}\right\|_{*}$ as a substitute of the operator norm. A bound on $\left\|X_{\star}\right\|_{*}$ is often available, see Subsection 6.1. we can use Lemma 4.4 to bound the distance to the solution for (MinFeasSDP). Moreover, based on this bound, we can also estimate $\epsilon_{p}, \delta_{p}$ for the solution $X_{\mathrm{obj}}$ of (MinObjSDP) before solving it.

D.1. Computable bounds on the operator norm. When no prior bound on $\left\|X_{\star}\right\|_{\mathrm{op}}$ is available, we can invoke Lemma D.2 in the following to estimate $\left\|X_{\star}\right\|_{\mathrm{op}}$ using any feasible point of (MinFeasSDP). However, to obtain a good estimate, we might need to first solve (MinFeasSDP).

Lemma D.2. Suppose (P) and (D) admit solutions and satisfy strong duality. Let $S$ be feasible for (MinFeasSDP). Define $\epsilon, T$ as in Theorem D.1 and $\kappa_{V}=$ $\frac{\sigma_{\max }(\mathcal{A})}{\sigma_{\min }\left(\mathcal{A}_{V}\right)}$. Define the scaled distance bound $\phi=\left(1+\kappa_{V}\right) \sqrt{\frac{\epsilon}{T}}$ and the infeasibility $\delta_{S}=\left\|\mathcal{A}_{V}(S)-b\right\|_{2}$. If $\sigma_{\min }\left(\mathcal{A}_{V}\right)>0 T>0$. Then $\left\|X_{\star}\right\|_{\mathrm{op}} \leq B$ for some constant $B$, where

$$
B=\frac{1}{4}\left[\sqrt{2 \phi^{2}+4\left(\frac{\delta_{S}}{\sigma_{\min }\left(\mathcal{A}_{V}\right)}+\|S\|_{\mathrm{op}}\right)+4 \frac{\phi}{1+\kappa_{V}}}+\sqrt{2} \phi\right]^{2} .
$$

Proof. Use inequality (4.3) in Lemma 4.4 to see $\left\|S-S_{\star}\right\|_{\mathrm{F}} \leq \frac{\delta_{S}}{\sigma_{\min }\left(\mathcal{A}_{V}\right)}$ for a minimizer $S_{\star}$ of (MinFeasSDP). Combine this with (4.2) in Lemma 4.4 to obtain

$$
\left\|V S V^{\top}-X_{\star}\right\|_{\mathrm{F}} \leq\left(1+\kappa_{V}\right)\left(\frac{\epsilon}{T}+\sqrt{2 \frac{\epsilon}{T}\left\|X_{\star}\right\|_{\mathrm{op}}}\right)+\frac{\delta_{S}}{\sigma_{\min }\left(\mathcal{A}_{V}\right)} .
$$

Because $\left\|V S V^{\top}-X_{\star}\right\|_{\mathrm{op}} \geq\|X\|_{\mathrm{op}}-\|S\|_{\mathrm{op}}$, we further have

$$
\left\|X_{\star}\right\|_{\mathrm{op}}-\|S\|_{\mathrm{op}} \leq\left(1+\kappa_{V}\right)\left(\frac{\epsilon}{T}+\sqrt{2 \frac{\epsilon}{T}\left\|X_{\star}\right\|_{\mathrm{op}}}\right)+\frac{\delta_{S}}{\sigma_{\min }\left(\mathcal{A}_{V}\right)} .
$$

Solve the above inequality for $\left\|X_{\star}\right\|_{\text {op }}$ to find a formula for the bound $B$.

The quantities $T, V$ appearing in Theorem D.1 can all be computed from available information. The problem is evaluating $\sigma_{\max }(\mathcal{A})$ appearing in $\kappa_{V}$, which might require evaluating $\mathcal{A}$ on full $n \times n$ matrices. Of course, It might be possible to know $\sigma_{\max }(\mathcal{A})$ in priori if we have some structure information of it. 
D.2. A few words on well-posedness. Theorem D.1 makes no guarantee on the quality of the reconstructed primal when $\min \left\{\sigma\left(\mathcal{A}_{V}\right), T\right\}=0$. In fact, this failure signals either that $y$ is far from optimality, or that the primal (P) or dual (D) is degenerate (violating the assumptions from Subsection 1.1).

To see this, suppose for simplicity, we know the rank $r=r_{\star}$ of a solution to (P). If $y$ is close to $y_{\star}$ and the primal (P) and dual (D) are degenerate, then Lemma 4.5 shows that the quantities $\min \left\{\sigma\left(\mathcal{A}_{V}\right), T\right\}$ are close to $\min \left\{\sigma\left(\mathcal{A}_{V_{\star}}\right), \lambda_{n-r_{\star}}\left(Z\left(y_{\star}\right)\right)\right\}$. Furthermore, Lemma 4.5 shows that our assumptions (from Subsection 1.1) guarantee $\min \left\{\sigma\left(\mathcal{A}_{V_{\star}}\right), \lambda_{n-r_{\star}}\left(Z\left(y_{\star}\right)\right)\right\}>0$. Thus if $\min \left\{\sigma\left(\mathcal{A}_{V}\right), T\right\}=0$, then either we need a more accurate solution to the dual problem to recover the primal, or the problem is degenerate and our assumptions fail to hold.

Appendix E. Lemmas for fixing infeasible dual iterates in Section 6. We present one lemma to bound the infeasibility of a dual vector $y$, and another to show how to construct a feasible $y$ from an infeasible one.

Lemma E.1. Suppose (P) and (D) admit solutions and satisfy strong duality, Equation (1.1). Let $\underline{\alpha}:=\inf _{X \in \mathcal{X}_{\star}} \operatorname{tr}(X)$ where $\mathcal{X}_{\star}$ is the solution set of $(\mathrm{P})$. For any dual vector $y$ with suboptimality $\operatorname{tr}\left(C X_{\star}\right)-g_{\alpha}(y) \leq \epsilon$ with $\alpha>\underline{\alpha}$, we have $\lambda_{\min }(Z(y)) \geq-\frac{\epsilon}{\alpha-\underline{\alpha}}$.

This lemma shows infeasibility decreases at the same speed as suboptimality.

Proof. Let $Z=C-\mathcal{A}^{\top} y$. Assume $\lambda_{\min }(Z)<0$. (Otherwise, we are done.) Then for any $X_{\star} \in \mathcal{X}_{\star}$

$$
\begin{aligned}
\operatorname{tr}\left(C X_{\star}\right)-g_{\alpha}(y) & =\operatorname{tr}\left(C X_{\star}\right)-b^{\top} y-\alpha \lambda_{\min }(Z) \\
& =\operatorname{tr}\left(C X_{\star}\right)-\left(\mathcal{A} X_{\star}\right)^{\top} y-\alpha \lambda_{\min }(Z) \\
& =\operatorname{tr}\left(Z X_{\star}\right)-\alpha \lambda_{\min }(Z) .
\end{aligned}
$$

Using the suboptimality assumption and Von Neumann's inequality, we further have

$$
\epsilon \geq \operatorname{tr}\left(Z X_{\star}\right)-\alpha \lambda_{\min }(Z) \geq \operatorname{tr}\left(X_{\star}\right) \lambda_{\min }(Z)-\alpha \lambda_{\min }(Z) .
$$

Rearrange to see $\lambda_{\min }(Z) \geq-\frac{\epsilon}{\alpha-\operatorname{tr}\left(X_{\star}\right)}$. Let $\operatorname{tr}\left(X_{\star}\right) \rightarrow \underline{\alpha}$ to obtain the result.

We next show how to construct an $\epsilon$-suboptimal and feasible dual vector from an $\epsilon$-suboptimal and potentially infeasible dual vector.

Lemma E.2. Suppose (P) and (D) admit solutions and satisfy strong duality, Equation (1.1). Further suppose a dual vector $y_{1}$ with $Z_{1}=C-\mathcal{A}^{\top} y_{1}$ is infeasible with $-\epsilon \leq \lambda_{\min }\left(Z_{1}\right)<0$ and $y_{2}$ with $Z_{2}=C-\mathcal{A}^{\top} y_{2}$ is strictly feasible in the sense that $\lambda_{\min }\left(Z_{2}\right)>0$, then the dual vector

$$
y_{\gamma}=\gamma y_{1}+(1-\gamma) y_{2}
$$

is feasible for $\gamma=\frac{\lambda_{\min }\left(Z_{2}\right)}{\epsilon+\lambda_{\min }\left(Z_{2}\right)}$. The objective value of $y_{\gamma}$ is

$$
g_{\alpha}\left(y_{\gamma}\right)=\frac{\lambda_{\min }\left(Z_{2}\right)}{\epsilon+\lambda_{\min }\left(Z_{2}\right)} b^{\top} y_{1}+\frac{\epsilon}{\epsilon+\lambda_{\min }\left(Z_{2}\right)} b^{\top} y_{2}
$$

Proof. The results follow from the linearity of $C-\mathcal{A}^{\top} y$ and the concavity of $\lambda_{\min }$.

Appendix F. Additional numerics. 
F.1. Primal Recovery for Matrix Completion. For matrix completion, we generate a random rank 5 matrix $\bar{X} \in \mathbf{R}^{1000 \times 1500}$. We generate the set $\Omega$ by observing each entry of $\bar{X}$ with probability 0.025 independently. To evaluate our method, we compare the recovered primal with $\bar{X}$, which (with high probability) solves the Matrix Completion problem [20]. The rest of the setting is exactly the same as in subsection 7.1. The plot is shown in and we come to the same conclusion as in subsection 7.1.
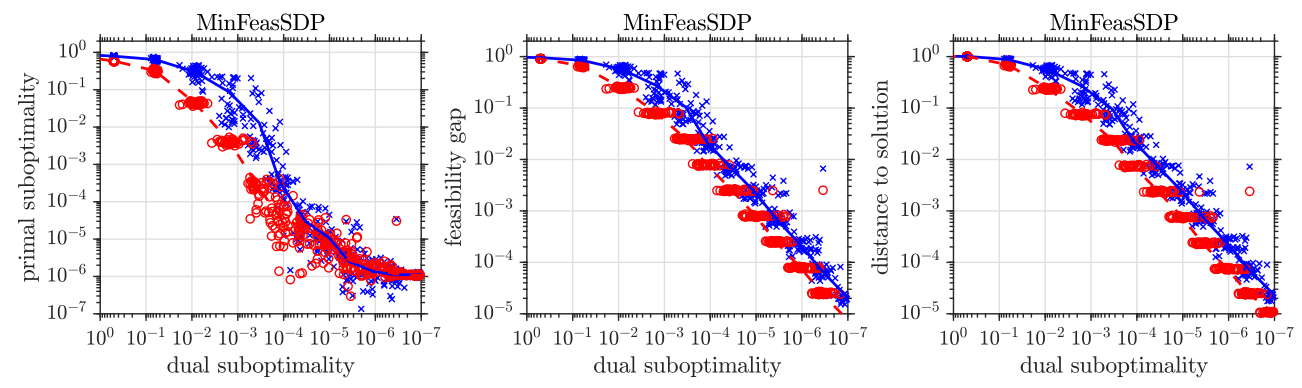

Fig. 4: (MinFeasSDP)
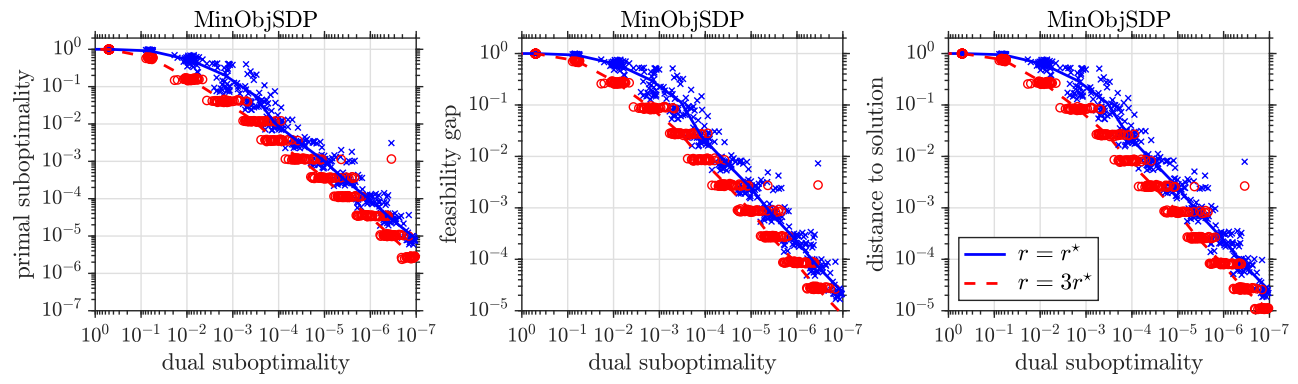

Fig. 5: (MinObjSDP)

Fig. 6: The plots shows the primal recovery performance of (MinFeasSDP) (upper) and (MinObjSDP) (lower) in terms of primal suboptimality, infeasibility and the distance to solution for the matrix completion problem. The horizontal axis is the dual suboptimality. The blue dots corresponds to the choice $r=r_{\star}$ and the red dots corresponds to the choice $r=3 r_{\star}$ in Algorithm 6.1.

F.2. Solving primal SDP with various dual solvers of medium scale problems. In this section we show that Algorithm 6.1 (Dual Algorithm + Primal Recovery) solves the primal SDP, using the dual solvers AdaGrad [27], AdaNGD [43], and AcceleGrad. Here we perform the primal recovery in every iteration of the dual algorithms. The problem instance for max-cut is the same as Subsection 7.1, and the instance for matrix completion is the same as Appendix F.1. Here we use (MinFeasSDP) to recover the primal. The numerical results are shown in Figure 9. We plot the relative dual suboptimality, primal suboptimality, infeasibility and distance to solution (as explained in subsection 7.1) for each iteration of the dual method. The solid lines show recovery with $r=r_{\star}$ while the dotted lines use the higher rank 
$r=3 r_{\star}$.

We observe convergence in each of these metrics, as expected from theory. Primal and dual suboptimality converge faster than the other two quantities, as in Figure 1. Interestingly, while AccelGrad converges much faster than the other algorithms on the dual side, its advantage on the primal side is more modest. We again see that the primal recovered using the larger rank $r=3 r_{\star}$ converges more quickly, though interestingly using the higher rank confers less of an advantage in reducing distance to the solution than in reducing primal suboptimality and infeasibility.
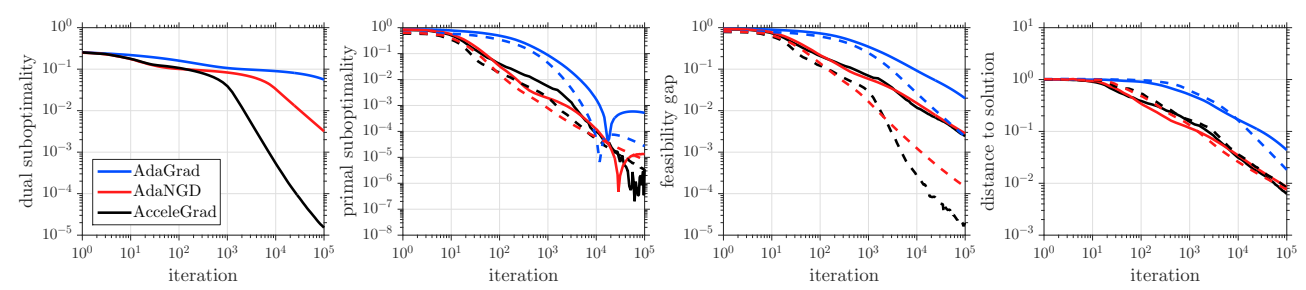

Fig. 7: Max-Cut
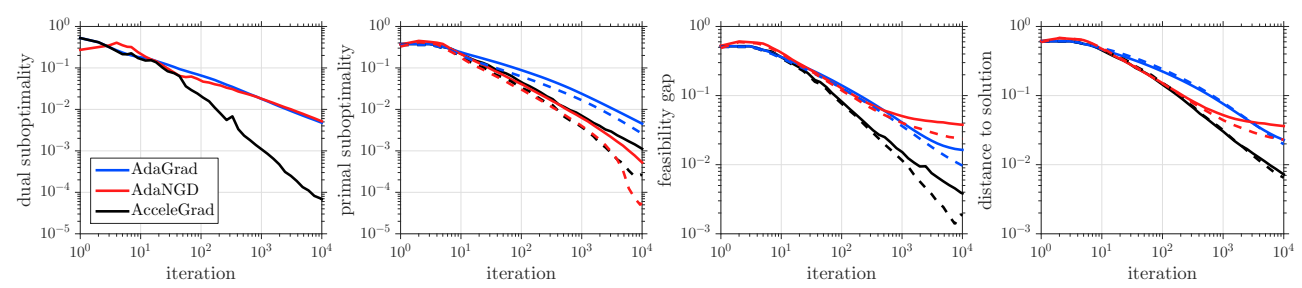

Fig. 8: Matrix Completion

Fig. 9: Plots from left to right columns show convergence of penalized dual objective $g_{\alpha}$, primal suboptimality, infeasibility, and distance to solution. The solid lines show recovery with $r=r_{\star}$ while the dotted lines use the higher rank $r=3 r_{\star}$.

F.3. Accuracy versus time and comparison to existing solvers. In this section, we present additional numerics regarding accuracy versus time to Subsection 7.3. We perform the same procedure as there to Max-Cut SDP for G1 $\left(800^{2}\right)$, G45 $\left(10^{3} \times 10^{3}\right)$ and G67 $\left(10^{4} \times 10^{4}\right)$ in the Gset, and G_n_pin_pout $\left(10^{5} \times 10^{5}\right)$ in the DIAMCS10 group. The results can be found in Figure 14. Results for a matrix completion problem, simulated as described in Subsection 7.2 with $c=1,10$, and 100, with decision variable size $\left(n_{1}+n_{2}\right)^{2}=(125 c)^{2}$ with $n_{1}=75 c$ and $n_{2}=50 c$, and $25\left(n_{1}+n_{2}\right) \log \left(n_{1}+n_{2}\right)$ are shown in Figure 18. We also compare with existing solver SDPNAL+ for medium scale problems: Max-Cut problem G45 and matrix completion with $n_{1}+n_{2}=1250$. We note our method achieves medium accuracy $10^{-3}$ in less than 100 seconds for medium scale problems. Such results are comparable or even better than SDPNAL+. 

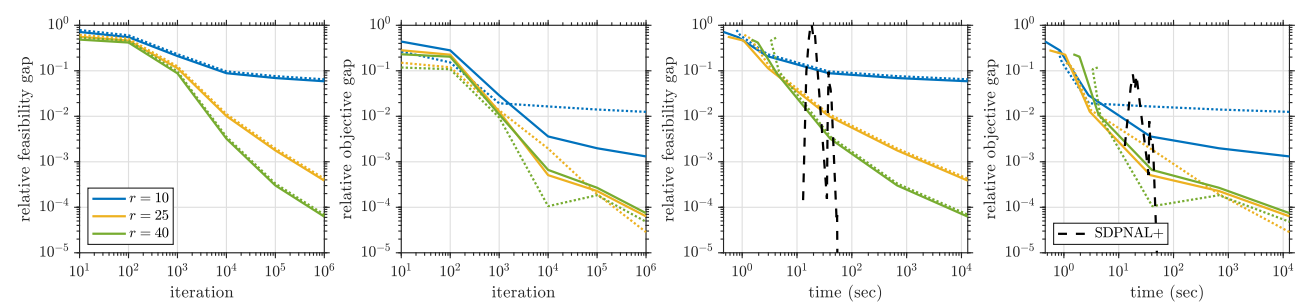

Fig. 10: Max-Cut: G1
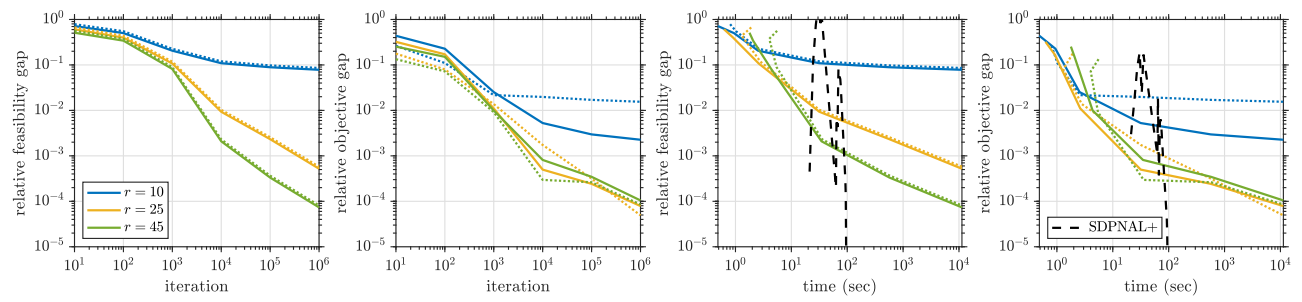

Fig. 11: Max-Cut: G45
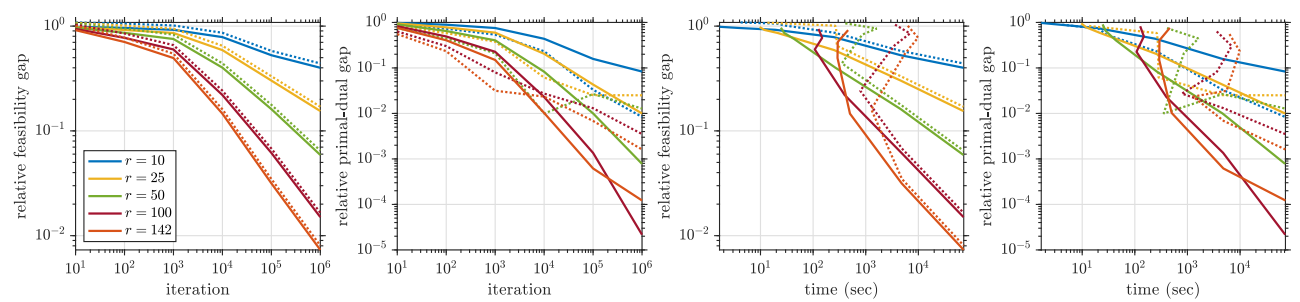

Fig. 12: Max-Cut: G75
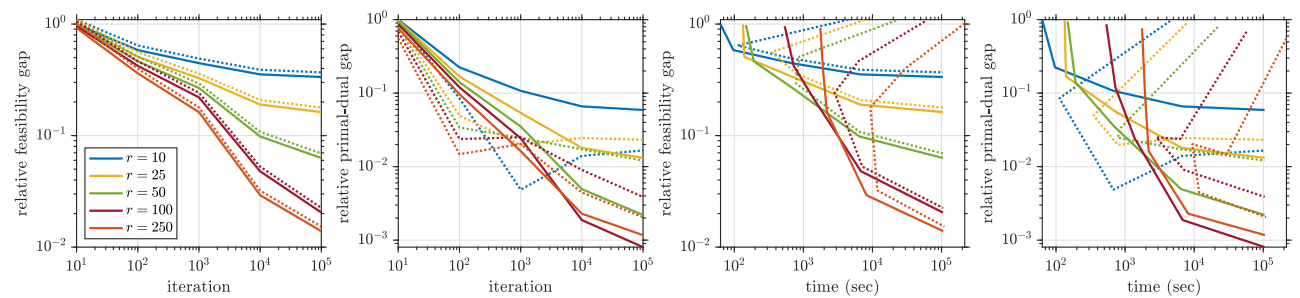

Fig. 13: Max-Cut: G_n_pin_pout

Fig. 14: Here we show the convergence of scaled suboptimality and infeasibility of our Algorithm 6.1 (option 1 as the solid line and option 2 as the dotted line.) against the actual time and iteration counter. 

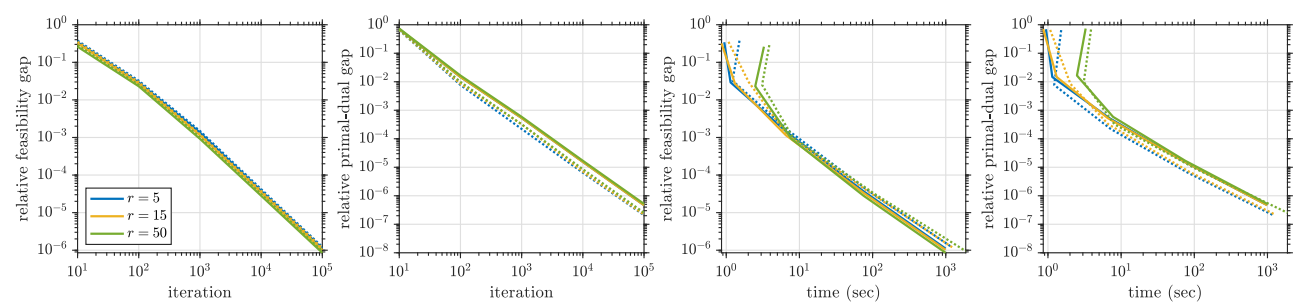

Fig. 15: $n_{1}=75, n_{2}=50$
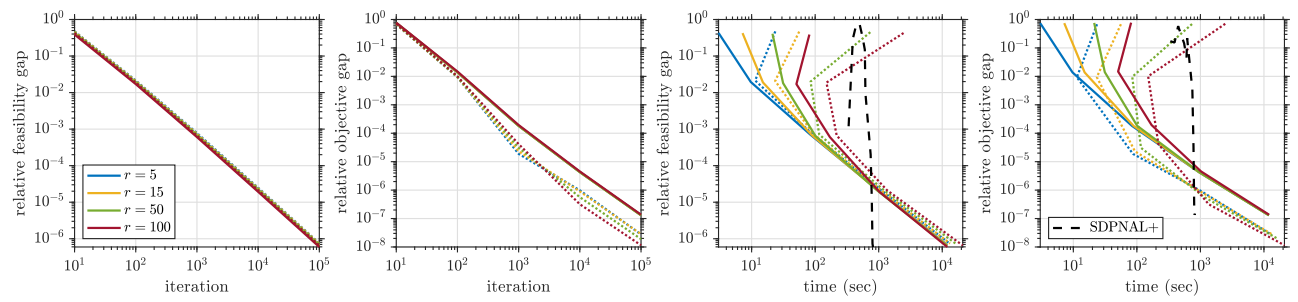

Fig. 16: $n_{1}=750, n_{2}=500$
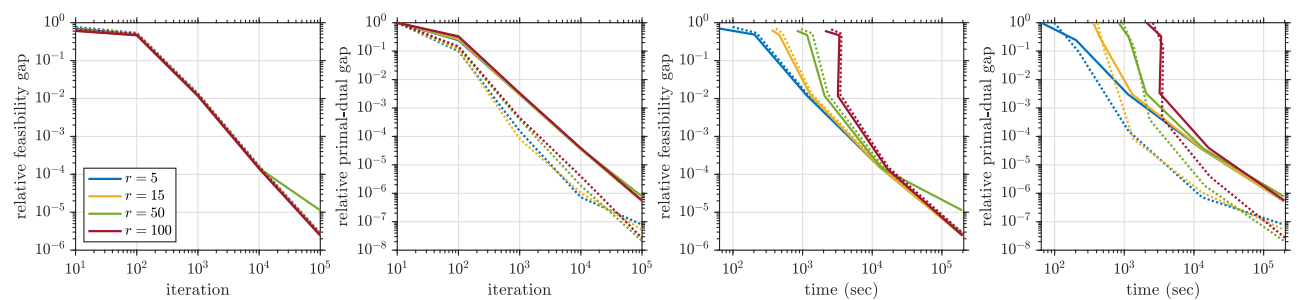

Fig. 17: $n_{1}=7500, n_{2}=5000$

Fig. 18: Here we show the convergence of scaled suboptimality and infeasibility of our Algorithm 6.1 (option 1 as the solid line and option 2 as the dotted line.) against the actual time and iteration counter.

Acknowledgments. Lijun Ding and Madeleine Udell were supported in part by DARPA Award FA8750-17-2-0101. Parts of this research were conducted while Madeleine Udell was in residence at the Simons Institute. Alp Yurtsever and Volkan Cevher have received funding for this project from the European Research Council (ERC) under the European Union's Horizon 2020 research and innovation programme (grant agreement no 725594-time-data), and from the Swiss National Science Foundation (SNSF) under grant number 200021_178865/1. Joel A. Tropp was supported in part by ONR Awards No. N-00014-11-1002, N-00014-17-12146, and N-00014-1812363.

\section{REFERENCES}

[1] The university of florida sparse matrix collection: Dimacs10 group, https://www.cise.ufl.edu/ research/sparse/matrices/DIMACS10/index.html.

[2] The university of florida sparse matrix collection: Gset group, https://www.cise.ufl.edu/ 
research/sparse/matrices/Gset/index.html (accessed 2015-06-10).

[3] F. Alizadeh, Combinatorial optimization with interior point methods and semi-definite matrices, Ph. D. thesis, University of Minnesota, (1991).

[4] F. AlizADEH, Interior point methods in semidefinite programming with applications to combinatorial optimization, SIAM journal on Optimization, 5 (1995), pp. 13-51.

[5] F. Alizadeh, J.-P. A. Haeberly, and M. L. Overton, Complementarity and nondegeneracy in semidefinite programming, Mathematical programming, 77 (1997), pp. 111-128.

[6] F. Alizadeh, J.-P. A. Haeberly, and M. L. Overton, Primal-dual interior-point methods for semidefinite programming: convergence rates, stability and numerical results, SIAM Journal on Optimization, 8 (1998), pp. 746-768.

[7] X. Bai, H. Wei, K. Fujisawa, and Y. Wang, Semidefinite programming for optimal power flow problems, International Journal of Electrical Power \& Energy Systems, 30 (2008), pp. 383-392.

[8] A. I. Barvinok, Problems of distance geometry and convex properties of quadratic maps, Discrete \& Computational Geometry, 13 (1995), pp. 189-202.

[9] A. Beck And M. TeBoulle, A fast iterative shrinkage-thresholding algorithm for linear inverse problems, SIAM journal on imaging sciences, 2 (2009), pp. 183-202.

[10] R. Bellman and K. Fan, On systems of linear inequalities in hermitian matrix variables, Convexity, 7 (1963), pp. 1-11.

[11] S. J. Benson, Y. Ye, AND X. Zhang, Solving large-scale sparse semidefinite programs for combinatorial optimization, SIAM Journal on Optimization, 10 (2000), pp. 443-461.

[12] N. Boumal, V. Voroninski, and A. Bandeira, The non-convex burer-monteiro approach works on smooth semidefinite programs, in Advances in Neural Information Processing Systems, 2016, pp. 2757-2765.

[13] S. Boyd, L. El Ghaoui, E. Feron, and V. Balakrishnan, Linear matrix inequalities in system and control theory, vol. 15, Siam, 1994.

[14] S. Boyd, N. Parikh, E. Chu, B. Peleato, J. Eckstein, et al., Distributed optimization and statistical learning via the alternating direction method of multipliers, Foundations and Trends $($ in Machine learning, 3 (2011), pp. 1-122.

[15] S. Boyd and L. Vandenberghe, Convex optimization, Cambridge university press, 2004.

[16] S. Burer And R. D. Monteiro, A nonlinear programming algorithm for solving semidefinite programs via low-rank factorization, Mathematical Programming, 95 (2003), pp. 329-357.

[17] S. Burer And R. D. Monteiro, Local minima and convergence in low-rank semidefinite programming, Mathematical Programming, 103 (2005), pp. 427-444.

[18] E. J. Candes, Y. C. Eldar, T. Strohmer, and V. Voroninski, Phase retrieval via matrix completion, SIAM review, 57 (2015), pp. 225-251.

[19] E. J. CANDÈs AND B. RECht, Exact matrix completion via convex optimization, Foundations of Computational mathematics, 9 (2009), p. 717.

[20] E. J. CANDÈs AND T. TAO, The power of convex relaxation: Near-optimal matrix completion, IEEE Transactions on Information Theory, 56 (2010), pp. 2053-2080.

[21] A. Chai, M. Moscoso, and G. Papanicolaou, Array imaging using intensity-only measurements, Inverse Problems, 27 (2010), p. 015005.

[22] A. Chambolle And T. Pock, A first-order primal-dual algorithm for convex problems with applications to imaging, Journal of mathematical imaging and vision, 40 (2011), pp. 120145.

[23] Y. Chen, Y. Chi, And A. J. Goldsmith, Exact and stable covariance estimation from quadratic sampling via convex programming, IEEE Transactions on Information Theory, 61 (2015), pp. 4034-4059.

[24] K. L. Clarkson, Coresets, sparse greedy approximation, and the frank-wolfe algorithm, ACM Transactions on Algorithms (TALG), 6 (2010), p. 63.

[25] S. Diamond And S. Boyd, Matrix-free convex optimization modeling, in Optimization and its applications in control and data sciences, Springer, 2016, pp. 221-264.

[26] D. Drusvyatskiy And A. S. Lewis, Error bounds, quadratic growth, and linear convergence of proximal methods, Mathematics of Operations Research, (2018).

[27] J. Duchi, E. Hazan, And Y. Singer, Adaptive subgradient methods for online learning and stochastic optimization, Journal of Machine Learning Research, 12 (2011), pp. 2121-2159.

[28] M. Frank and P. Wolfe, An algorithm for quadratic programming, Naval research logistics quarterly, 3 (1956), pp. 95-110.

[29] M. P. Friedlander AND I. MaCedo, Low-rank spectral optimization via gauge duality, SIAM Journal on Scientific Computing, 38 (2016), pp. A1616-A1638.

[30] D. Gabay AND B. Mercier, A dual algorithm for the solution of non linear variational problems via finite element approximation, Institut de recherche d'informatique et 
d'automatique, 1975.

[31] R. Glowinski and A. Marroco, Sur l'approximation, par éléments finis d'ordre un, et la résolution, par pénalisation-dualité d'une classe de problèmes de dirichlet non linéaires, Revue française d'automatique, informatique, recherche opérationnelle. Analyse numérique, 9 (1975), pp. 41-76.

[32] M. X. Goemans And D. P. Williamson, Improved approximation algorithms for maximum cut and satisfiability problems using semidefinite programming, Journal of the ACM (JACM), 42 (1995), pp. 1115-1145.

[33] N. Halko, P. G. Martinsson, and J. A. Tropp, Finding structure with randomness: probabilistic algorithms for constructing approximate matrix decompositions, SIAM Rev., 53 (2011), pp. 217-288.

[34] E. HAZAN, Sparse approximate solutions to semidefinite programs, in Latin American Symposium on Theoretical Informatics, Springer, 2008, pp. 306-316.

[35] C. Helmberg and F. Rende, A spectral bundle method for semidefinite programming, SIAM Journal on Optimization, 10 (2000), pp. 673-696.

[36] S. Homer and M. Peinado, Design and performance of parallel and distributed approximation algorithms for maxcut, Journal of Parallel and Distributed Computing, 46 (1997), pp. 4861.

[37] M. JAGGI, Revisiting frank-wolfe: Projection-free sparse convex optimization., in Proceedings of the 30th international conference on machine learning, no. CONF, 2013, pp. 427-435.

[38] P. R. Johnstone And P. Moulin, Faster subgradient methods for functions with $h \backslash$ ” olderian growth, arXiv preprint arXiv:1704.00196, (2017).

[39] R. H. Keshavan, A. Montanari, and S. OH, Matrix completion from a few entries, IEEE transactions on information theory, 56 (2010), pp. 2980-2998.

[40] J. KUCZYŃSKI AND H. WoŹNIAKOWSKI, Estimating the largest eigenvalue by the power and lanczos algorithms with a random start, SIAM journal on matrix analysis and applications, 13 (1992), pp. 1094-1122.

[41] A. Lemon, A. M.-C. So, Y. Ye, ET AL., Low-rank semidefinite programming: Theory and applications, Foundations and Trends $\AA$ in Optimization, 2 (2016), pp. 1-156.

[42] E. S. Levitin and B. T. Polyak, Constrained minimization methods, Zhurnal Vychislitel'noi Matematiki i Matematicheskoi Fiziki, 6 (1966), pp. 787-823.

[43] K. LEvy, Online to offline conversions, universality and adaptive minibatch sizes, in Advances in Neural Information Processing Systems, 2017, pp. 1613-1622.

[44] K. Y. Levy, A. Yurtsever, And V. Cevher, Online adaptive methods, universality and acceleration, arXiv preprint arXiv:1809.02864, (2018).

[45] D. Lijun and U. Madeleine, On the regularity and conditioning of low rank semidefinite programs.

[46] R. Madani, S. Sojoudi, and J. Lavaei, Convex relaxation for optimal power flow problem: Mesh networks, IEEE Transactions on Power Systems, 30 (2015), pp. 199-211.

[47] J. Malick, J. Povh, F. Rendl, and A. Wiegele, Regularization methods for semidefinite programming, SIAM Journal on Optimization, 20 (2009), pp. 336-356.

[48] C. Mathieu and W. Schudy, Correlation clustering with noisy input, in Proceedings of the twenty-first annual ACM-SIAM symposium on Discrete Algorithms, Society for Industrial and Applied Mathematics, 2010, pp. 712-728.

[49] L. Mirsky, A trace inequality of john von neumann, Monatshefte für mathematik, 79 (1975), pp. 303-306.

[50] A. Mosek, The mosek optimization software, Online at http://www. mosek. com, 54 (2010), p. 5 .

[51] Y. Nesterov, Introductory lectures on convex optimization: A basic course, vol. 87, Springer Science \& Business Media, 2013.

[52] Y. Nesterov And A. Nemirovski, Self-concordant functions and polynomial time methods in convex programming, ussr acad, Sci., Central Economic\&Mathematical Institute, Moscow, (1989).

[53] Y. Nesterov AND A. Nemirovskit, Interior-point polynomial algorithms in convex programming, vol. 13, Siam, 1994.

[54] B. O'Donoghue, E. Chu, N. Parikh, And S. Boyd, Conic optimization via operator splitting and homogeneous self-dual embedding, Journal of Optimization Theory and Applications, 169 (2016), pp. 1042-1068, http://stanford.edu/ boyd/papers/scs.html.

[55] B. O'Donoghue, E. Chu, N. Parikh, and S. Boyd, SCS: Splitting conic solver, version 2.0.2. https://github.com/cvxgrp/scs, Nov. 2017.

[56] G. PATAKI, On the rank of extreme matrices in semidefinite programs and the multiplicity of optimal eigenvalues, Mathematics of operations research, 23 (1998), pp. 339-358. 
[57] N. RAO, P. Shah, AND S. WRight, Conditional gradient with enhancement and truncation for atomic-norm regularization, in NIPS workshop on Greedy Algorithms, 2013.

[58] B. Recht, M. Fazel, AND P. A. PARRIlo, Guaranteed minimum-rank solutions of linear matrix equations via nuclear norm minimization, SIAM review, 52 (2010), pp. 471-501.

[59] J. RENEGAR, Efficient first-order methods for linear programming and semidefinite programming, arXiv preprint arXiv:1409.5832, (2014).

[60] R. T. RockafEllar, Monotone operators and the proximal point algorithm, SIAM journal on control and optimization, 14 (1976), pp. 877-898.

[61] A. P. Ruszczyński And A. Ruszczynski, Nonlinear optimization, vol. 13, Princeton university press, 2006.

[62] N. Srebro And A. Shraibman, Rank, trace-norm and max-norm, in International Conference on Computational Learning Theory, Springer, 2005, pp. 545-560.

[63] J. F. Sturm, Using sedumi 1.02, a matlab toolbox for optimization over symmetric cones, Optimization methods and software, 11 (1999), pp. 625-653.

[64] J. F. StuRm, Error bounds for linear matrix inequalities, SIAM Journal on Optimization, 10 (2000), pp. 1228-1248.

[65] D. Sun, K.-C. Toh, Y. Yuan, And X.-Y. Zhao, Sdpnalt: A matlab software for semidefinite programming with bound constraints (version 1.0), Optimization Methods and Software, 35 (2020), pp. 87-115.

[66] M. J. TodD, Semidefinite optimization, Acta Numerica, 10 (2001), pp. 515-560.

[67] K.-C. TOH, M. J. TODD, AND R. H. TüTüNC̈̈, Sdpt3a matlab software package for semidefinite programming, version 1.3, Optimization methods and software, 11 (1999), pp. 545-581.

[68] L. Vandenberghe and S. Boyd, Semidefinite programming, SIAM review, 38 (1996), pp. 4995.

[69] I. Waldspurger, A. DAspremont, and S. Mallat, Phase recovery, maxcut and complex semidefinite programming, Mathematical Programming, 149 (2015), pp. 47-81.

[70] I. Waldspurger And A. WATERS, Rank optimality for the burer-monteiro factorization, arXiv preprint arXiv:1812.03046, (2018).

[71] Z. Wen, D. GoldFARB, AND W. YIN, Alternating direction augmented lagrangian methods for semidefinite programming, Mathematical Programming Computation, 2 (2010), pp. 203230.

[72] Y. Yu, T. Wang, And R. J. Samworth, A useful variant of the davis-kahan theorem for statisticians, Biometrika, 102 (2014), pp. 315-323.

[73] A. Yurtsever, O. Fercoq, and V. Cevher, A conditional gradient-based augmented lagrangian framework, arXiv preprint arXiv:1901.04013, (2019).

[74] A. Yurtsever, O. Fercoq, F. Lochtello, and V. Cevher, A conditional gradient framework for composite convex minimization with applications to semidefinite programming, arXiv preprint arXiv:1804.08544, (2018).

[75] A. Yurtsever, Y.-P. Hsien, AND V. Cevher, Scalable convex methods for phase retrieval, in Computational Advances in Multi-Sensor Adaptive Processing (CAMSAP), 2015 IEEE 6th International Workshop on, IEEE, 2015, pp. 381-384.

[76] A. Yurtsever, M. Udell, J. Tropp, and V. Cevher, Sketchy decisions: Convex low-rank matrix optimization with optimal storage, in Artificial Intelligence and Statistics, 2017, pp. 1188-1196.

[77] X.-Y. ZhaO, D. Sun, AND K.-C. TOH, A newton-cg augmented lagrangian method for semidefinite programming, SIAM Journal on Optimization, 20 (2010), pp. 1737-1765. 